\title{
WIND STRUCTURE AND LUMINOSITY VARIATIONS IN THE WOLF-RAYET/LUMINOUS BLUE VARIABLE HD 5980*
}

\author{
LeONid GeOrgiev $^{1,8}$, Gloria Koenigsberger ${ }^{2}$, D. John Hillier ${ }^{3}$, Nidia Morrell ${ }^{4}$, \\ RODOLFO BARBÁ ${ }^{5,6}$, AND ROBERTO GAMEN ${ }^{7}$ \\ ${ }^{1}$ Instituto de Astronomía, Universidad Nacional Autónoma de México, Apdo. Postal 70-264, México D.F., 04510, Mexico; georgiev@astro.unam.mx \\ ${ }^{2}$ Instituto de Ciencias Físicas, Universidad Nacional Autónoma de México, Apdo. Postal 48-3, Cuernavaca, Mor. 62210, Mexico; gloria@ astro.unam.mx \\ ${ }^{3}$ Department of Astronomy, 3941 O'Hara Street, University of Pittsburgh, Pittsburgh, PA 15260, USA \\ ${ }^{4}$ Las Campanas Observatory, The Carnegie Observatories, Colina El Pino s/n, Casillas 601, La Serena, Chile \\ ${ }^{5}$ Departamento de Física, Universidad de la Serena, Benavente 980, La Serena, Chile \\ ${ }^{6}$ ICATE-CONICET, San Juan, Argentina \\ ${ }^{7}$ Facultad de Ciencias Astronómicas y Geofísicas, Universidad Nacional de La Plata, and Instituto de Astrofísica de La Plata (CCT La Plata-CONICET), \\ Paseo del Bosque S/N, B1900FWA, La Plata, Argentina \\ Received 2011 July 4; accepted 2011 September 16; published 2011 November 7
}

\begin{abstract}
Over the past 40 years, the massive luminous blue variable/Wolf-Rayet system HD 5980 in the Small Magellanic Cloud (SMC) has undergone a long-term S Doradus-type variability cycle and two brief and violent eruptions in 1993 and 1994. In this paper we analyze a collection of UV and optical spectra obtained between 1979 and 2009 and perform CMFGEN model fits to spectra of 1994, 2000, 2002, and 2009. The results are as follows: (1) the long-term S Dor-type variability is associated with changes of the hydrostatic radius; (2) the 1994 eruption involved changes in its bolometric luminosity and wind structure; (3) the emission-line strength, the wind velocity, and the continuum luminosity underwent correlated variations in the sense that a decreasing $V_{\infty}$ is associated with increasing emission line and continuum levels; and (4) the spectrum of the third star in the system $(\operatorname{Star} C)$ is well fit by a $T_{\text {eff }}=32 \mathrm{~K}$ model atmosphere with SMC chemical abundances. For all epochs, the wind of the erupting star is optically thick at the sonic point and is thus driven mainly by the continuum opacity. We speculate that the wind switches between two stable regimes driven by the "hot" (during the eruption) and the "cool" (post-eruption) iron opacity bumps as defined by Lamers \& Nugis and Gräfener \& Hamann, and thus the wind may undergo a bi-stability jump of a different nature from that which occurs in OB stars.
\end{abstract}

Key words: binaries: eclipsing - stars: individual (HD 5980) - stars: variables: S Doradus - stars: Wolf-Rayet

Online-only material: color figures

\section{INTRODUCTION}

Eruptive mass-loss phenomenon in massive stars is emerging as an area of interest for many reasons, one of the most important of which is that the characteristics of certain supernova (SN) explosions depend critically on the progenitor's mass. The stars classified as luminous blue variables (LBVs) appear to have the ability to remove a large fraction of their outer stellar layers through violent ejection processes long before the $\mathrm{SN}$ phase is reached. It is believed that these ejections, combined with the effects of the stationary stellar winds, may determine to a large extent the mass of the SN progenitor (van Marle et al. 2007; Smith 2008; Dwarkadas 2011).

The LBV eruptions have been observed since the 1600 s, but the mechanisms driving the instability have not been identified. Although some scenarios have been suggested (Guzik et al. 2005; Owocki \& van Marle 2008; Kashi \& Soker 2010), their confirmation is difficult due to the large distances at which most of these objects lie and the fact that they are intensely observed only after they have undergone an eruptive event. Thus, their preeruption characteristics are poorly constrained. Furthermore, observational constraints on their fundamental parameters are often lacking.

\footnotetext{
* Based on data obtained with HST, IUE, FUSE, and the $6.5 \mathrm{~m}$ Magellan telescopes at Las Campanas Observatory in Chile.

8 Visiting Astronomer, Department of Astronomy, 3941 O'Hara Street, University of Pittsburgh, Pittsburgh, PA 15260, USA.
}

HD 5980 is a multiple system in the Small Magellanic Cloud (SMC) that has been observed spectroscopically since the 1960s. It contains an eclipsing binary system plus a third object. Four well-defined variability timescales are present: (1) a long-term ( $\sim 40$ years) variation (Koenigsberger et al. 2010); (2) sudden eruptive events which were observed in 1993 and 1994, each lasting less than 1 year (Barbá \& Niemela 1994; Barbá et al. 1995; Koenigsberger et al. 1995; Jones \& Sterken 1997); (3) orbital-phase locked variations with the 19.3 day eclipsing binary period (Breysacher et al. 1982; Foellmi et al. 2008, and references therein); and (4) "microvariability" on $\sim 30$ minute timescale that was observed shortly after the 1993/1994 eruptions (Breysacher 1997; Sterken \& Breysacher 1997). These timescales disclose the presence of a variety of physical phenomena in the system. In particular, Koenigsberger et al. (2010) have argued that the 1993-1994 eruptions may have been caused by tidal interactions that became stronger due to a gradual increase in the stellar radius, on the $\sim 40$ year timescale. Although the process responsible for the $\sim 40$ year timescale is unknown, it is believed to be the same as that which occurs in $\mathrm{S}$ Doradus-type variables.

The three luminous objects comprising the HD 5980 system are: two emission-line stars in a close 19.3 day binary orbit (Breysacher \& Perrier 1980) and a third O-type object (hereafter $\operatorname{Star} C$ ) whose photospheric absorptions remain relatively stationary on the orbital timescale of the binary (Niemela 1988; Koenigsberger et al. 2002). Following the convention introduced by Barbá et al. (1996), we label as Star A the star "in front" at 
orbital phase $\phi=0.00$ and Star B the one "in front" at the opposite eclipse, which occurs at $\phi=0.36$. Star $A$ is the unstable star of the system (Barbá et al. 1996), and whose spectral type has changed from the Wolf-Rayet (WR) subtype WN3 (in 1978-1981; Niemela 1988) to WN6 (in 1990; Koenigsberger et al. 1994) culminating in WN11/B1.5 $\mathrm{Ia}^{+}$during the 1994 eruptive phase (Drissen et al. 2001; Heydari-Malayeri et al. 1997; Koenigsberger et al. 1998a). Star B is believed to be a WN4 star (Breysacher et al. 1982; Niemela 1988). Further background on HD 5980's observational characteristics may be found in Barbá et al. (1996, 1997), Moffat et al. (1998), Koenigsberger (2004), and Foellmi et al. (2008).

Although its triple nature implies having to deal with the problem of disentangling the spectra of three stars and the possible contribution from a wind-wind interaction region to arrive at the eruptor's properties, HD 5980 provides considerable advantages for studying the underlying instability: (1) its distance and masses can be relatively well constrained; (2) it is un-obscured by dust; (3) it has been widely studied spectroscopically at X-ray, UV, and optical wavelengths; and (4) it was well observed during the stages prior to the 1993-1994 eruptions and intensely observed thereafter. In this paper we focus on determining the epoch-dependent properties of Star A. Section 2 is devoted to a description of the observational data; in Section 3 we empirically disentangle the wind velocities of the three stars in the system; the existence of correlations between visual magnitude, UV brightness, emission-line strength, and wind speed are shown to exist in Section 4. Section 5 contains the description of the model atmosphere fits to the spectra; the results are discussed in Section 6 and the conclusions presented in Section 7.

\section{OBSERVATIONAL MATERIAL}

Ultraviolet spectroscopy ( $\lambda \lambda 1190-2000)$ of HD 5980 is available from the International Ultraviolet Explorer (IUE) at low and high resolutions in 1979, 1981, 1989, 1991, and 1994-1995, and at low resolution in 1978 and 1986. The properties of these spectra were analyzed by Moffat et al. (1998) and Koenigsberger et al. (1994, 1995, 1996, 1998a, 1998b), and an overview of the derived results may be found by Koenigsberger (2004). Further UV observations at high resolution were obtained using the HST/Space Telescope Imaging Spectrograph (STIS) in 1999, 2000, 2002, and 2009. The properties of these spectra are described by Koenigsberger et al. (2000, 2001, 2010). Tables 1 and 2 list in the first three columns the identifying number for each spectrum, the date of acquisition and the corresponding orbital phase computed with the Sterken \& Breysacher (1997) initial epoch and orbital period. For many of the IUE spectra of HD 5980 a visual magnitude $m_{v}$ could be derived (Koenigsberger et al. 1994) from the Fine Error Sensor (FES) counts through the FES- $m_{v}$ calibrations (Perez 1992). These are listed in Column 4 of Tables 1 and 2. The FES magnitudes have a formal error of $0.07 \mathrm{mag}$.

The IUE spectra analyzed in this paper were retrieved from the Multimission Archive at STScI (MAST) database, which contains spectra that have been reprocessed with the Final Calibration. We find that the difference between the flux measurements made on these spectra and on the original data products are $<5 \%$ for spectra obtained prior to 1992 , but this difference increases significantly thereafter, in some cases being as high as $15 \% .^{9}$

\footnotetext{
9 As described by Nichols \& Linsky (1996), there were systematic wavelength-dependent discrepancies of up to $20 \%$ of the absolute flux calibration in some of the IUE spectra processed with previous software.
}

Velocities of spectral features were measured using consistent criteria for all data sets. Columns 5 and 6 of Table 1 list the velocities of selected features that will be described in the next section. We find that the uncertainty in the speeds measured for the P Cygni absorption components is generally $\sim 100 \mathrm{~km} \mathrm{~s}^{-1}$, which we adopt throughout this paper unless otherwise noted. The sources of uncertainty include the small signal-to-noise ratio $(\mathrm{S} / \mathrm{N})$ of $I U E$ spectra, the contamination by lines arising from other atomic transitions, and the definition of the continuum level. In general, the new velocity measurements coincide well, within the uncertainties, with those published previously.

The continuum flux at $\lambda 1850 \AA$ was measured on all UV spectra and is listed in Columns 9 and 7 of Tables 1 and 2 , respectively. The $\lambda 1850 \AA$ spectral region was chosen to characterize the UV continuum level because it is relatively line-free in most of the HD 5980 spectra. The integrated flux of the N IV] $1486 \AA$ and N III $1750 \AA$ blend was measured and the values are listed in Columns 7 and 8 of Table 1 and 5 and 6 of Table 2 . Both lines are clearly associated with the active state of Star A. N IV] $1486 \AA$ was first seen in 1986 , being absent or very weak previously. The N III $1750 \AA$ emission was first seen during the declining phase of the 1994 eruption. ${ }^{10}$ It appeared as a single emission line in low-resolution spectra but was composed of $\mathrm{N}$ III $2 s^{2} 2 p-2 s 2 p^{2}$ and $\mathrm{N}$ III $2 s 2 p^{2}-2 p^{3}$ multiplets. The flux contained in the N IV] $1486 \AA$ and N III emission lines was measured on the low dispersion spectra by fitting one or more Gaussian functions. On high dispersion spectra, the flux was obtained through direct integration over the emission feature. The largest source of uncertainty resides in the choice of the continuum level which is frequently difficult to define due to large number of variable emission lines and, on high-resolution IUE spectra, the low $\mathrm{S} / \mathrm{N}$.

The formal uncertainties in the flux calibrations of $I U E$ and STIS data are, respectively, $\sim 6 \%$ (Colina et al. 1996) and $1 \%$ (Maíz-Apellaniz \& Bohlin 2005). In general, the flux levels measured in high- and low-resolution IUE spectra differ by no more than the quoted uncertainty. However, in the subset of observations obtained in 1994, the high-resolution data display significantly weaker flux levels than the contemporaneous lowresolution spectra. This situation might be due to tracking problems during the long exposures. Also, the FES counts are not available during this time period. ${ }^{11}$ Hence, for the analysis described in the following sections, we measured the continuum flux only on low-resolution IUE data for the 1994 epoch.

An optical spectrum was obtained on 2009 November 7, at phase 0.038 using the Magellan Echellette (MagE) on the Clay $6.5 \mathrm{~m}$ (Magellan-II) telescope on Las Campanas. We used the $1^{\prime \prime}$ slit providing a spectral resolution of $1 \AA$. Thirteen echelle orders were extracted covering the wavelength region from $3130 \mathrm{~A}$ to $9400 \AA$. The $\mathrm{S} / \mathrm{N}$ ranges from 100 to 200 for a single $150 \mathrm{~s}$ exposure. The usual ThAr comparison lamp was used for wavelength calibration. The data were reduced using the special set of IRAF routines available for the reduction of Magellan Inamori Kyosera Echelle (MIKE) spectra (mtools package; http://www.lco.cl/telescopes-information/ magellan/instruments/mike/iraf-tools). Spectra of the standards Feige 110 and NGC 7293 central star observed during the same night were used to derive a sensitivity function. The individual

\footnotetext{
10 There are unfortunately no UV spectra obtained earlier during this event.

11 No report of FES malfunction was found in the IUE Operations Summary, although a change in the Gyro 5 drift rate is reported to have occurred in 1994 October as well as the appearance of the Data Multiplexer Unit (DMU) anomaly; see http://wise-iue.tau.ac.il/ines/docs/p05.pdf.
} 
Table 1

High Dispersion Spectra

\begin{tabular}{|c|c|c|c|c|c|c|c|c|}
\hline Spectrum & $\mathrm{HJD}^{\mathrm{a}}$ & Phase & FES Mag & He II edge ${ }^{b}$ & $\mathrm{NV}_{\mathrm{sat}}{ }^{\mathrm{c}}$ & $\mathrm{F}(\mathrm{N} \text { IV })^{\mathrm{d}}$ & $\mathrm{F}\left(\mathrm{N}_{\text {III }}\right)^{\mathrm{d}}$ & $\mathrm{F}(1850 \AA)^{\mathrm{e}}$ \\
\hline 4277 & 43921.1 & 0.57 & 11.68 & -2770 & -2800 & $<1$. & 1.0 & 1.47 \\
\hline 4345 & 43928.6 & 0.91 & 11.69 & -2620 & -2760 & $<1$. & $<1$ & 1.53 \\
\hline \multirow[t]{2}{*}{4958} & 43981.0 & 0.68 & 11.72 & -2800 & -2050 & $<1$. & $<1$ & 1.43 \\
\hline & $\cdots$ & $\cdots$ & $\ldots$ & $\cdots$ & -2790 & $\ldots$ & $\cdots$ & \\
\hline 11175 & 44632.3 & 0.48 & 11.61 & -2920 & -2610 & 2.8 & 2.7 & 1.58 \\
\hline 11190 & 44634.6 & 0.60 & 11.63 & -2920 & -2540 & $<1.3$ & $<1$ & 1.64 \\
\hline 15072 & 44869.5 & 0.80 & 11.62 & -2740 & -2450 & 1.9: & $<1$ & 1.61 \\
\hline \multirow[t]{2}{*}{15080} & 44870.5 & 0.85 & 11.57 & -2730 & -2130 & 0.7: & 1.5 & 1.28 \\
\hline & $\cdots$ & $\cdots$ & $\cdots$ & $\ldots$ & -2650 & $\cdots$ & $\ldots$ & \\
\hline 37759 & 47867.2 & 0.39 & 11.36 & $\begin{array}{l}-2520 \\
-2080^{\mathrm{f}}\end{array}$ & -2210 & 11.2 & $\begin{array}{l}1 . \\
\ldots\end{array}$ & 1.59 \\
\hline 37768 & 47868.2 & 0.44 & 11.27 & -2550 & -2050 & 10.7 & $<1$ & 1.62 \\
\hline 37781 & $\begin{array}{c}\ldots \\
47870.2\end{array}$ & $\begin{array}{c}\cdots \\
0.55\end{array}$ & $\begin{array}{c}\ldots \\
11.27\end{array}$ & $-1890^{\mathrm{f}}$ & $\begin{array}{c}\cdots \\
-1750\end{array}$ & $\ldots$ & $\ldots$ & $\cdots$ \\
\hline 37788 & 47871.4 & 0.61 & 11.25 & -2000 & -1950 & 143 & 02 & 185 \\
\hline 42446 & 48511.5 & 0.83 & 11.41 & -1900 & -1980 & 12.5 & $<1$ & 1.86 \\
\hline 42470 & 48515.6 & 0.05 & 11.35 & -1700 & -1800 & 16.9 & $<1$ & 1.82 \\
\hline 42694 & 48541.4 & 0.39 & 11.55 & -2650 & -2450 & 13.2 & 1. & 1.73 \\
\hline 42702 & 48542.4 & 0.44 & 11.50 & -2480 & -2340 & 11.1 & 2. & 1.86 \\
\hline 42711 & 48543.4 & 0.49 & 11.27 & -2480 & -2210 & 14.5 & 1.4 & 1.93 \\
\hline 42721 & 48544.4 & 0.54 & 11.33 & -2450 & $-2180^{\mathrm{g}}$ & 17.8 & $<1$ & 1.95 \\
\hline 52888 & 49680.5 & 0.51 & 11.37 & -420 & $\ldots$ & 7.4 & 18.8 & 3.32 \\
\hline $53036^{\mathrm{h}}$ & 49697.5 & 0.39 & $\ldots$ & -370 & $\ldots$ & 14.0: & 39.1: & $1.96::$ \\
\hline $53129^{\mathrm{h}}$ & 49706.5 & 0.86 & $\ldots$ & -470 & $\ldots$ & 6.9: & 34.6: & $1.75::$ \\
\hline $53216^{\mathrm{h}}$ & 49716.5 & 0.38 & $\ldots$ & -370 & -1680 & 23.9 & 57.0 & 2.55: \\
\hline $53226^{\mathrm{h}}$ & 49717.5 & 0.43 & $11.12^{\mathrm{i}}$ & -370 & -1730 & 21.9 & 58.1 & 2.55: \\
\hline 54064 & 49784.1 & 0.89 & $\ldots$ & -1100 & -2010 & 38.8 & 39.3 & 2.50 \\
\hline \multirow{2}{*}{54490} & 49831.0 & 0.32 & 11.96 & $-800^{\mathrm{f}}$ & $\ldots$ & 43.6 & 35.9 & 2.65 \\
\hline & $\ldots$ & & $\cdots$ & -1900 & -1660 & - & $\ldots$ & $\ldots$ \\
\hline \multirow[t]{2}{*}{54671} & 49851.0 & 0.36 & 11.98 & $-810^{\mathrm{f}}$ & -950 : & 49.0 & 35.7 & 2.49 \\
\hline & & & & -2000 & -1900 & & ... & \\
\hline \multirow[t]{2}{*}{54727} & 49859.9 & 0.82 & 11.96 & $-1100^{f}$ & -900 & 37.0 & 31.6 & 2.81 \\
\hline & $\ldots$ & & & -2400 & -2300 & & $\ldots$ & \\
\hline \multirow[t]{2}{*}{55315} & 49916.8 & 0.78 & 11.27 & $-1100^{\mathrm{f}}$ & $-800:$ & 35.9 & 20.1 & 2.55 \\
\hline & & & & -2530 & -2150 & & & \\
\hline \multirow{2}{*}{55380} & 49928.8 & 0.40 & 11.34 & $-900^{\mathrm{f}}$ & -1130 & 38.2 & 19.2 & 2.36 \\
\hline & & & & -2230 & -2400 & & & \\
\hline \multirow[t]{2}{*}{55394} & 49930.8 & 0.507 & 11.22 & $-900^{\mathrm{f}}$ & $-900:$ & 38.6 & 18.6 & 2.38 \\
\hline & 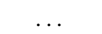 & & & -2380 & -2180 & & $\ldots$ & \\
\hline \multirow[t]{2}{*}{55932} & 49975.6 & 0.83 & 11.22 & $-1200^{f}$ & -1000 & 31.9 & 14.7 & 2.43 \\
\hline & $\ldots$ & & & -2620 & -2400 : & & $\ldots$ & \\
\hline \multirow[t]{2}{*}{55955} & 49978.7 & 0.99 & 11.37 & $-1200^{\mathrm{f}}$ & -880 : & 31.9 & 14.4 & 1.98 \\
\hline & & & & -2530 & -2330 & & $\ldots$ & \\
\hline 56017 & 49986.6 & 0.40 & 11.31 & -2670 & -2560 & 37.4 & 14.4 & 2.15 \\
\hline 56188 & 50033.5 & 0.83 & $\ldots$ & -1400 & -1850 & 29.0 & 10.6 & 2.29 \\
\hline \multirow[t]{2}{*}{56205} & 50036.4 & 0.99 & $\ldots$ & -1500 & -1000 & 31.9 & 8.2 & 1.76 \\
\hline & & & $\ldots$ & & -2160 & & & \\
\hline 56223 & 50045.2 & 0.43 & $\ldots$ & -2530 & -2500 & 30.7 & 7.1 & 2.15 \\
\hline \multirow[t]{2}{*}{1070} & 51304.8 & 0.83 & $\ldots$ & $-1770:$ & -1600 & 24.9 & 0.8 & 2.08 \\
\hline & & & $\ldots$ & $\cdots$ & -2050 & & & \\
\hline 3070 & 51308.9 & 0.05 & $\ldots$ & -1380 & -1570 & 25.0 & 0.4 & 1.68 \\
\hline 4070 & 51310.8 & 0.15 & $\ldots$ & -1350 & $-1670^{f}$ & 25.0 & 0.6 & 2.15 \\
\hline \multirow[t]{2}{*}{5070} & 51314.9 & 0.36 & $\ldots$ & -2500 & -1630 & 23.6 & 0.5 & 1.67 \\
\hline & $\cdots$ & & $\ldots$ & $\cdots$ & $-2130^{\mathrm{j}}$ & & $\ldots$ & \\
\hline 6070 & 51315.8 & 0.40 & $\ldots$ & -2140 & -1650 & 26.4 & 0.2 & 1.85 \\
\hline & & & $\cdots$ & $\cdots$ & -2140 & & & \\
\hline 2070 & 51655.1 & 0.00 & $\ldots$ & -1500 & $-1830^{f}$ & 17.9 & 0.9 & 1.68 \\
\hline 7070 & 52386.6 & 0.99 & $\mathrm{k}$ & -1860 & -2100 & 12.2 & $\ldots$ & \\
\hline 8070 & 55083.9 & 0.99 & $11.6^{1}$ & -2260 & -2460 & 7.0 & $<0.2$ & 1.50 \\
\hline & $\ldots$ & $\ldots$ & $\ldots$ & -2610 & $\ldots$ & $\ldots$ & $\ldots$ & $\ldots$ \\
\hline
\end{tabular}

Notes.

${ }^{\text {a }}$ Heliocentric Julian Date -2400000 .

${ }^{\mathrm{b}}$ Location where P Cyg absorption intersects the continuum level in $\mathrm{km} \mathrm{s}^{-1}$. Corrected for an adopted SMC systemic velocity of $+150 \mathrm{~km} \mathrm{~s}{ }^{-1}$.

c Maximum extent of the plateau ("flat" region) of P Cyg absorption in $\mathrm{km} \mathrm{s}^{-1}$; two values in this column indicate the presence of a second plateau at a different intensity level. Corrected for an adopted SMC systemic velocity of $+150 \mathrm{~km} \mathrm{~s}^{-1}$.

${ }^{\mathrm{d}}$ Integrated un-dereddened absolute flux in units of $10^{-12} \mathrm{erg} \mathrm{cm}^{-2} \mathrm{~s}^{-1}$.

e Un-dereddened absolute flux in units of $10^{-12} \mathrm{erg} \mathrm{cm}^{-2} \mathrm{~s}^{-1} \AA^{-1}$.

${ }^{\mathrm{f}}$ Maximum extent of "plateau."

g The absorption is not flat, but contains considerable structure.

${ }^{\text {h }}$ Flux calibration problem.

${ }^{\mathrm{i}}$ Visual magnitude from Koenigsberger et al. 1998b.

j Speed of what may be interpreted as a short plateau; absorption continues to rise gradually out $-3100 \mathrm{~km} \mathrm{~s}^{-1}$.

${ }^{\mathrm{k}}$ Visual magnitude of 11.3 for this same epoch but out of eclipse was provided by S. Dufau \& M. T. Ruiz (2002, private communication).

${ }^{1}$ All Sky Automated Survey (ASAS; Pojmanski 2002) value obtained $\sim 3 \mathrm{hr}$ earlier. 
Table 2

Low-resolution IUE Spectra

\begin{tabular}{|c|c|c|c|c|c|c|}
\hline SWP & $\mathrm{HJD}^{\mathrm{a}}$ & Phase & FES Mag & $\left.F\left(\mathrm{~N}_{\text {IV }}\right]\right)^{\mathrm{b}}$ & $F(\mathrm{~N} \text { III })^{\mathrm{b}}$ & $F(1850 \AA)^{\mathrm{c}}$ \\
\hline 1598 & 43650.5 & 0.53 & 11.72 & 1.8 & 6.52 & 1.61 \\
\hline 14112 & 44754.4 & 0.82 & 11.61 & 1.8 & 2.8 & 1.73 \\
\hline 14135 & 44756.2 & 0.92 & 11.63 & 5.1 & 0.7 & 1.67 \\
\hline 14166 & 44758.8 & 0.05 & 11.56 & 4.6 & 1.6 & 1.68 \\
\hline 29633 & 46743.7 & 0.07 & 11.42 & 5.3 & 0 & 1.69 \\
\hline 29673 & 46748.9 & 0.34 & 11.59 & 8.9 & 2 & 1.35 \\
\hline 29674 & 46748.9 & 0.35 & 11.57 & 7.9 & 2 & 1.39 \\
\hline 29681 & 46749.7 & 0.39 & 11.49 & 6.1 & 2 & 1.52 \\
\hline 29690 & 46750.8 & 0.45 & 11.44 & 14.7 & 2 & 1.68 \\
\hline 29693 & 46750.9 & 0.45 & 11.45 & 8.2 & 2 & 1.75 \\
\hline 29699 & 46751.8 & 0.51 & 11.41 & 8.2 & 0 & 1.76 \\
\hline 29702 & 46751.9 & 0.51 & 11.39 & 5.0 & 1 & 1.75 \\
\hline 29705 & 46752.7 & 0.54 & 11.41 & 7.7 & 2 & 1.77 \\
\hline 29708 & 46752.8 & 0.55 & 11.39 & 5.3 & 1.5 & 1.76 \\
\hline 29736 & 46757.9 & 0.81 & 11.37 & 5.9 & 2 & 1.67 \\
\hline 29743 & 46758.7 & 0.85 & 11.40 & 7.3 & 2 & 1.69 \\
\hline 37760 & 47867.5 & 0.39 & 11.30 & 11.7 & 2.0 & 1.81 \\
\hline 37769 & 47868.5 & 0.44 & 11.25 & 12.8 & 1 & 1.89 \\
\hline 37782 & 47870.5 & 0.56 & 11.22 & 14.0 & 1 & 1.93 \\
\hline 37789 & 47871.5 & 0.61 & 11.20 & 14.3 & 1 & 1.96 \\
\hline 37790 & 47871.5 & 0.61 & 11.37 & 18.6 & 3 & 1.91 \\
\hline 42445 & 48511.5 & 0.83 & 11.26 & 18.7 & 0.9 & 1.99 \\
\hline 42469 & 48515.5 & 0.04 & 11.43 & 16.9 & 0.5 & 1.82 \\
\hline 42693 & 48541.4 & 0.38 & 12.00 & 12.9 & 1 & 1.73 \\
\hline 42701 & 48542.4 & 0.44 & 11.39 & 13.7 & 2 & 1.98 \\
\hline 42703 & 48542.7 & 0.45 & 11.49 & 17.2 & 0.4 & 2.01 \\
\hline 42710 & 48543.4 & 0.49 & 11.27 & 18.3 & 2.8 & 2.06 \\
\hline 42720 & 48544.4 & 0.54 & 11.26 & 14.0 & 1. & 2.04 \\
\hline 42722 & 48544.7 & 0.55 & 11.33 & 16.1 & 1.7 & 2.09 \\
\hline 52824 & 49674.9 & 0.22 & 10.11 & 4.0 & 26.5 & 3.50 \\
\hline 52889 & 49680.6 & 0.52 & 10.19 & 1. & 27.2 & 3.50 \\
\hline 52922 & 49684.8 & 0.73 & 10.10 & 4 & 33.6 & 3.10 \\
\hline 52956 & 49688.6 & 0.93 & 10.22 & 0.8 & 29.3 & 3.05 \\
\hline 52967 & 49689.7 & 0.99 & $\ldots$ & 10.8 & 37.4 & 3.17 \\
\hline 52975 & 49690.7 & 0.04 & $\ldots$ & 12.1 & 38.3 & 3.08 \\
\hline 52992 & 49692.7 & 0.14 & 10.54 & 12.0 & 41.8 & 2.74 \\
\hline 53035 & 49697.2 & 0.38 & $\ldots$ & 23.1 & 42.6 & 2.65 \\
\hline 53037 & 49697.6 & 0.40 & $\ldots$ & 15.4 & 44.1 & 2.60 \\
\hline 53061 & 49702.6 & 0.66 & $\ldots$ & 17.1 & 52.3 & 2.65 \\
\hline 53128 & 49706.3 & 0.85 & $\ldots$ & 18.6 & 45.0 & 2.72 \\
\hline 53164 & 49709.9 & 0.03 & $\ldots$ & 18.1 & 45.8 & 2.65 \\
\hline 53186 & 49712.6 & 0.18 & $\ldots$ & 30.7 & 51.5 & 2.80 \\
\hline 53187 & 49712.7 & 0.18 & $\ldots$ & 31.1 & 53.3 & 2.84 \\
\hline 53218 & 49716.6 & 0.39 & $\ldots$ & 34.6 & 56.2 & 2.90 \\
\hline 53230 & 49717.8 & 0.44 & $\ldots$ & 36.5 & 59.8 & 3.12 \\
\hline 54485 & 49830.3 & 0.28 & $\ldots$ & 45.8 & 37.1 & 2.89 \\
\hline 54486 & 49830.5 & 0.29 & $\ldots$ & 40.2 & 37.7 & 2.94 \\
\hline 54491 & 49831.2 & 0.33 & $\ldots$ & 43.9 & 38.2 & 2.65 \\
\hline 54533 & 49836.3 & 0.59 & $\ldots$ & 39.8 & 38.3 & 3.24 \\
\hline 54534 & 49836.3 & 0.59 & $\ldots$ & 43.3 & 42.7 & 3.30 \\
\hline 54535 & 49836.3 & 0.59 & $\ldots$ & 43.4 & 41.9 & 3.23 \\
\hline 54664 & 49850.3 & 0.32 & $\ldots$ & 41.9 & 38.6 & 2.62 \\
\hline 54665 & 49850.4 & 0.33 & $\ldots$ & 42.1 & 38.1 & 2.68 \\
\hline 54666 & 49850.4 & 0.33 & $\ldots$ & 46.4 & 36.1 & 2.70 \\
\hline 54670 & 49850.8 & 0.35 & $\ldots$ & 47.4 & 39.5 & 2.61 \\
\hline 54708 & 49857.4 & 0.69 & $\ldots$ & 44.0 & 37.8 & 2.85 \\
\hline 54709 & 49857.4 & 0.69 & $\ldots$ & 40.4 & 37.2 & 2.93 \\
\hline 54728 & 49860.1 & 0.83 & $\ldots$ & 45.5 & 30.1 & 2.93 \\
\hline 54758 & 49864.1 & 0.04 & $\ldots$ & 37.7 & 34.0 & 2.53 \\
\hline 54759 & 49864.2 & 0.04 & $\ldots$ & 35.1 & 34.4 & 2.37 \\
\hline 54760 & 49864.2 & 0.04 & $\ldots$ & 32.6 & 37.8 & 2.26 \\
\hline 55111 & 49894.1 & 0.59 & 10.94 & 41.6 & 27.0 & 2.60 \\
\hline 55112 & 49894.1 & 0.60 & 10.21 & 45.3 & 29.5 & 2.65 \\
\hline 55314 & 49916.7 & 0.77 & 11.27 & 39.3 & 20.4 & 2.69 \\
\hline 55340 & 49921.0 & 0.99 & $\ldots$ & 41.2 & 23.8 & 2.33 \\
\hline 55381 & 49928.9 & 0.40 & 11.30 & 42.0 & 20.9 & 2.38 \\
\hline
\end{tabular}

Table 2

(Continued)

\begin{tabular}{ccccccc}
\hline \hline SWP & HJD $^{\mathrm{a}}$ & Phase & FES Mag & $F(\mathrm{~N} \text { IV })^{\mathrm{b}}$ & $F(\mathrm{~N} \mathrm{III})^{\mathrm{b}}$ & $F(1850 \AA)^{\mathrm{c}}$ \\
\hline 55382 & 49928.9 & 0.51 & $\ldots$ & 40.2 & 21.6 & 2.48 \\
55382 & 49928.9 & 0.51 & $\ldots$ & 40.2 & 21.6 & 2.48 \\
55395 & 49930.9 & 0.51 & 11.19 & 42.5 & 24.1 & 2.60 \\
55396 & 49930.9 & 0.51 & 11.25 & 40.3 & 22.1 & 2.55 \\
55461 & 49939.9 & 0.97 & 11.33 & 43.7 & 24.2 & 2.17 \\
55462 & 49939.9 & 0.98 & $\ldots$ & 39.0 & 18.3 & 2.13 \\
55931 & 49975.5 & 0.82 & $\ldots$ & 45.5 & 13.0 & 2.48 \\
55933 & 49975.8 & 0.84 & $\ldots$ & 40.4 & 15.3 & 2.40 \\
55954 & 49978.8 & 0.99 & $\ldots$ & 41.9 & 19.0 & 1.96 \\
55956 & 49978.8 & 0.99 & $\ldots$ & 36.8 & 14.9 & 2.14 \\
55957 & 49978.8 & 0.00 & $\ldots$ & 37.1 & 14.6 & 2.07 \\
55958 & 49978.9 & 0.00 & $\ldots$ & 35.5 & 18.6 & 2.08 \\
55976 & 49981.8 & 0.15 & $\ldots$ & 33.0 & 15.8 & 2.40 \\
55977 & 49981.9 & 0.15 & $\ldots$ & 39.2 & 18.1 & 2.48 \\
56005 & 49985.0 & 0.32 & $\ldots$ & 42.7 & 17.3 & 2.03 \\
56006 & 49985.1 & 0.33 & $\ldots$ & 40.0 & 13.9 & 2.20 \\
56013 & 49985.9 & 0.36 & $\ldots$ & 37.2 & 16.3 & 2.17 \\
56014 & 49985.9 & 0.36 & $\ldots$ & 36.7 & 13.0 & 2.08 \\
56015 & 49985.9 & 0.36 & $\ldots$ & 32.0 & 13.2 & 2.13 \\
56018 & 49986.7 & 0.40 & $\ldots$ & 42.2 & 15.3 & 2.34 \\
56033 & 49990.8 & 0.62 & $\ldots$ & 38.7 & 15.3 & 2.48 \\
56034 & 49990.9 & 0.62 & $\ldots$ & 31.7 & 14.3 & 2.47 \\
\hline
\end{tabular}

Notes.

${ }^{\text {a }}$ Heliocentric Julian Date -2400000 .

b Integrated un-dereddened absolute flux in units of $10^{-12} \mathrm{erg} \mathrm{cm}^{-2} \mathrm{~s}^{-1}$.

${ }^{c}$ Un-dereddened absolute flux in units of $10^{-12} \mathrm{erg} \mathrm{cm}^{-2} \mathrm{~s}^{-1} \AA^{-1}$.

flux calibrated echelle orders were then normalized and merged in the final spectrum.

For the modeling discussed below, four representative spectra corresponding to different observation epochs were constructed as follows.

Spectrum 1994. The low-resolution IUE spectra SWP 53218 and LWP 29794 were combined with the optical spectrum obtained on the same date, 1994 December 30, at CTIO and described in Koenigsberger et al. (1998b). This combined spectrum covers the wavelength region from $1190 \AA$ to $6900 \AA$. The orbital phase is 0.39 , and this is the only spectrum not obtained at primary eclipse $(\phi=0)$ that we analyze. During this epoch, the spectrum of Star A was dominant and, as in Koenigsberger et al. (1998b), the contribution from Star B is neglected.

Spectrum 2000. The HST/STIS spectrum obtained on 2000 April 20 at phase 0.00 . This spectrum covers the spectral range 1150-10800 ̊.

Spectrum 2002. The HST/STIS observation of 2002 April 4, obtained at phase 0.99 was combined with the Far-Ultraviolet Spectroscopic Explorer (FUSE) spectrum P2230101 taken on 2002 July 27 at phase 0.00 . The systematic long-term variations in HD 5980 were relatively small between 2002 and 2009, justifying that we combine these two spectra obtained $\sim 3$ months apart.

Spectrum 2009. The HST/STIS spectrum secured on 2009 September 9 at $\phi=0.99$ was combined with the Magellan-II optical spectrum obtained on 2009 November 7 at phase 0.04 . As in the previous case, the long-term variability is not expected to be significant. Of greater concern is the fact that the optical spectrum was obtained 0.05 in phase later than the UV spectrum. At $\phi=0.04$ the eclipse of Star B's continuum-emitting region is partial (see light curve in Foellmi et al. 2008). 

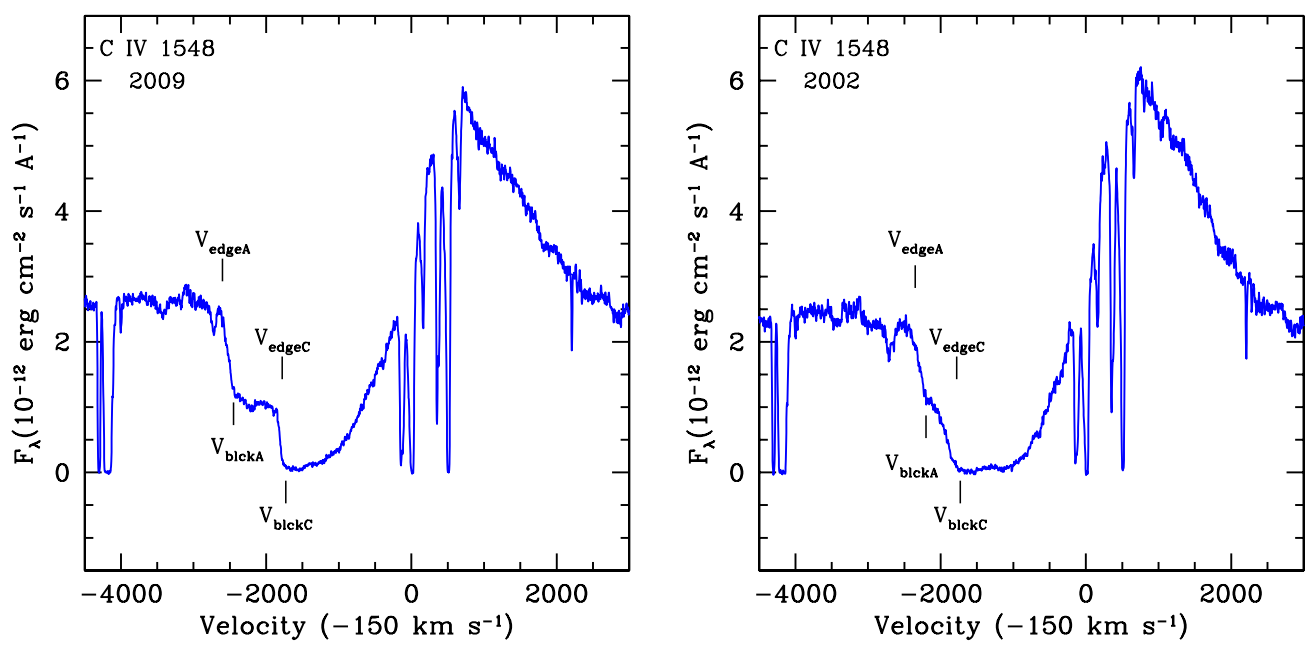

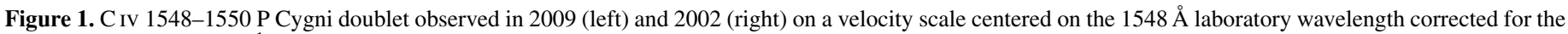
SMC motion $\left(+150 \mathrm{~km} \mathrm{~s}^{-1}\right)$.

(A color version of this figure is available in the online journal.)

\section{DISENTANGLING THE WIND VELOCITIES}

The terminal wind speed $V_{\infty}$ is generally derived from the saturated portion of the P Cygni absorption component, i.e., where the intensity reaches zero level, generally referred to as $V_{\text {black }}$ (Prinja et al. 1990). In the case of a binary system, the maximum extent of the saturated profile corresponds to the speed of the slower wind in the system. The faster wind also has a $V_{\text {black }}$, but the minimum intensity level lies at the continuum level of the star whose wind is slower. Thus, as shown in Georgiev \& Koenigsberger (2004), the absorption profile presents a steplike appearance. We will refer to the second $V_{\text {black }}$ as $V_{\text {plateau }}$. For single WR stars, it is generally found that for saturated lines $V_{\text {black }} \sim 0.70 V_{\text {edge }}$ (Prinja et al. 1990; Eenens \& Williams $1994)$, where $V_{\text {edge }}$ is the location where the P Cygni absorption meets the continuum level. The observation that $V_{\text {edge }}>V_{\text {black }}$ is generally attributed to an unspecified type of "turbulence" or to non-monotonicity in the wind. In the case of optically thin lines, the P Cygni absorptions do not reach the zero flux level. In this case, $V_{\text {edge }}$ provides information on the maximum wind speed attained within the particular line-forming region.

Interpreting the P Cygni absorption components in HD 5980 is difficult because 3 massive and hot stars contribute to its spectrum. Koenigsberger et al. (1998a) adopted the purely empirical approach of measuring $V_{\text {black }}$ and $V_{\text {edge }}$ in the IUE spectra obtained over the years 1979-1995. They found a persistent component at $V_{\text {black }} \sim-1740 \mathrm{~km} \mathrm{~s}^{-1}$ indicating the presence of a stable wind with this speed and showed that the wind speed of Star A had undergone changes from $<500 \mathrm{~km} \mathrm{~s}^{-1}$ to $\sim 1600 \mathrm{~km} \mathrm{~s}^{-1}$ during and after the 1994 eruption. However, although they suspected that Star A's wind speed had been as high as $\sim 3000 \mathrm{~km} \mathrm{~s}^{-1}$ in 1979 , disentangling its contribution to the P Cygni absorptions from that of the other two stars proved very challenging.

The more recent data have now clarified the picture, partly because of its greatly improved quality and partly because it has been possible to obtain UV observations during the orbital phase $\phi=0.0$, when Star A occults Star B. The size of the eclipsing disk of Star $A$ is a factor of 1.5 larger than Star B at minimum brightness (Perrier et al. 2009) and significantly larger during its more active state. It is therefore valid to assume that at orbital phases $\phi \sim 0.0$ Star A eclipses Star B's continuum-forming disk as well as the P Cyg absorption-forming region of Star B's wind. Thus, the P Cygni absorptions at $\phi \sim 0.0$ provide the wind speeds of Star A and Star C, without contamination from Star $B$.

\subsection{Star C: the "Third" Object}

Figure 1 is a plot of the C IV $1548 / 1550 \AA$ doublet observed in the HST/STIS spectra of 2009 (left panel) and 2002 (right panel) at orbital phase 0.99. A clear "step" is observed in the 2009 spectrum, providing two velocity values: $V_{\text {black }}=$ $-1760 \mathrm{~km} \mathrm{~s}^{-1}$ and $V_{\text {plateau }}=-2440 \mathrm{~km} \mathrm{~s}^{-1} .12$ The "step" is not as pronounced in the spectrum of 2002 (Figure 1, right), but still two velocity values may be measured: $V_{\text {black }}=-1780$ and $V_{\text {plateau }}=-2210 \mathrm{~km} \mathrm{~s}^{-1} \cdot V_{\text {black }}=-1770 \pm 10 \mathrm{~km} \mathrm{~s}^{-1}$ is consistent with the persistent wind velocity measured by Koenigsberger et al. (1998a) for all epochs prior to 1996. Since only two stars are visible at $\phi=0.99$, it is now clear that this stable component is formed in Star $C$. These spectra also give us the wind speed of Star A in 2002 and 2009, a topic to which we shall return in Section 3.3.

\subsection{Star B: the Elusive Companion}

Of the three distinguishable objects in HD 5980, Star B is the most elusive. From the $19.3 \mathrm{~d}$ radial velocity variations and the eclipse light curve, there is no question that Star B is a hot and massive object. However, given that Star $A$ is such a prominent source of emission-lines, it is difficult to determine the fraction of the emission-line spectrum that may be attributed to the wind of Star B. On the other hand, during the eclipse when Star B is "in front," the wind region of Star A where the fast portions of its P Cygni absorption components are formed is occulted. This eclipse occurs at $\phi=0.36$ and can be used to estimate Star B's wind speed.

In 1999, five HST/STIS spectra of HD 5980 were obtained over a single orbital cycle (Koenigsberger et al. 2000), including a spectrum at $\phi=0.36$. The line-profile of C IV 1548-50 A at this phase is shown in Figure 2 (right) where we observe $V_{\text {black }}=-1720 \mathrm{~km} \mathrm{~s}^{-1}$, associated with Star $C$ as discussed in

\footnotetext{
12 Note that the separation between these two $V_{\infty}$ values is $\sim 700 \mathrm{~km} \mathrm{~s}^{-1}$, significantly greater than the separation between the two $C$ IV doublets $\left(500 \mathrm{~km} \mathrm{~s}^{-1}\right)$.
} 

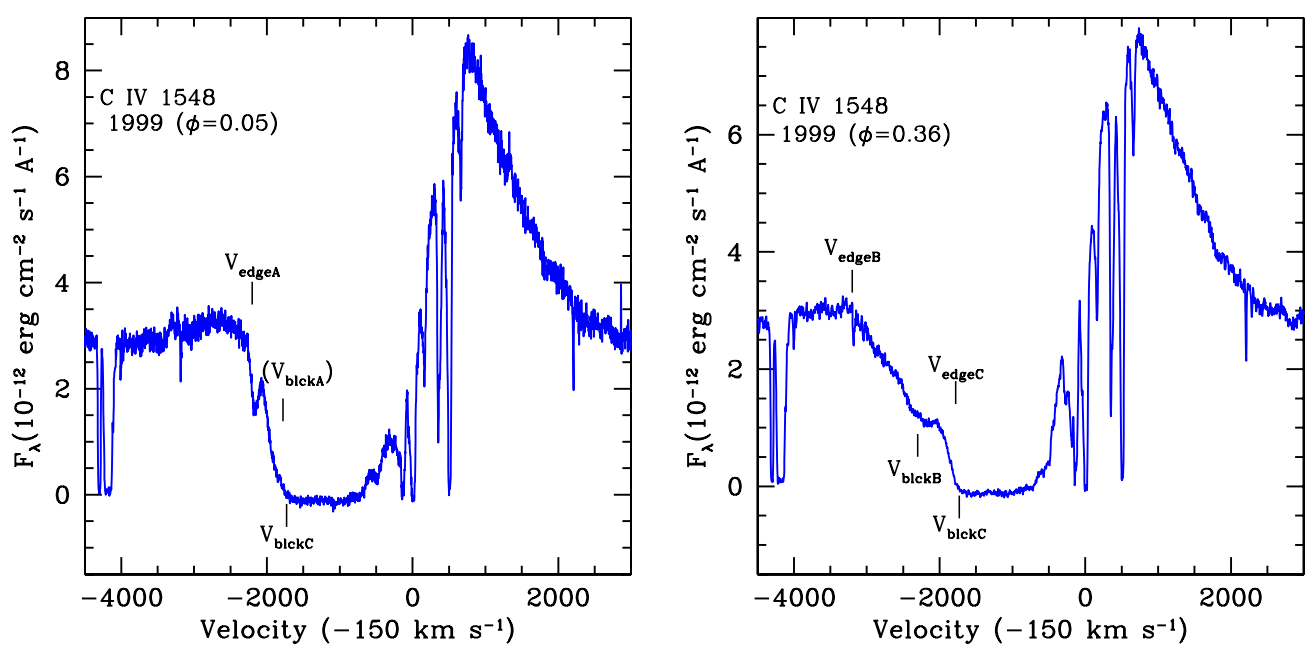

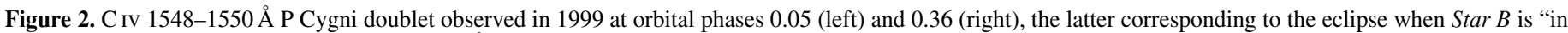

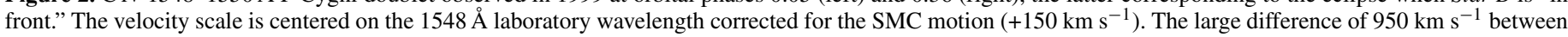
$V_{\text {edgeB }}$ and $V_{\text {blckB }}$ in right panel indicates a large amount of "turbulence."

(A color version of this figure is available in the online journal.)

the previous section. In addition, there is a short plateau that ends at $V_{\text {plateau }}=-2300 \pm 100 \mathrm{~km} \mathrm{~s}^{-1}$, followed by a very extended slope over which the line rises towards the continuum level extending to $V_{\text {edge }}=3100 \mathrm{~km} \mathrm{~s}^{-1}$. We associate the $V_{\text {plateau }}$ with the terminal wind speed of Star $B$. The value of $V_{\text {edge }}$ is consistent with the relation $V_{\text {black }} / V_{\text {edge }} \sim 0.76$ typically observed for WR stars (Prinja et al. 1990). It is important to note that the eclipse at $\phi=0.36$ is not total, since Star B's radius is smaller than that of Star A. However, the wind velocity component along the line of sight from the unocculted portion of Star A is rather slow and any P Cygni absorption formed in this star lies close to the rest-frame velocity. Thus, the very large value of $V_{\text {edge }}$ is most likely associated with "turbulence" in the wind of Star B.

At other orbital phases the picture is not as clear, however. Two observations at elongations were obtained as part of the same 1999 set of $H S T /$ STIS spectra mentioned above. The elongation phases observed are $\phi=0.15$ and $\phi=0.83$, when Star $B$ is approaching the observer and receding, respectively. At $\phi=0.15$ both the $-2300 \mathrm{~km} \mathrm{~s}^{-1}$ plateau and $-3100 \mathrm{~km} \mathrm{~s}^{-1}$ extended absorption should be clearly visible. However, the spectrum shows only $V_{\text {edge }} \sim-2400 \mathrm{~km} \mathrm{~s}^{-1}$, as illustrated in Figure 3. This suggests that the "turbulent" component is suppressed in the regions that are viewed expanding along the line of sight to the observer at $\phi=0.15$. This may be a consequence of the presence of the wind-wind interaction region, an issue that will be addressed in a forthcoming paper. For the present investigation, it suffices to keep in mind that Star $B$ 's wind velocity is $V_{B} \sim 2300 \mathrm{~km} \mathrm{~s}^{-1}$ and that its contribution to the system's spectrum is important only when it is "in front" of Star A.

\subsection{Star A: the Eruptor}

In Figure 2 (left) we illustrate the spectrum obtained in 1999 at $\phi=0.05$. This phase is close to primary eclipse, and Star A is between us and Star B. Here there is no plateau in the absorption line-profile indicating that Star A's terminal wind velocity is similar to that of $\operatorname{Star} C$ and both stars contribute to $V_{\text {black }}=-1720 \mathrm{~km} \mathrm{~s}^{-1}$. In Section 3.1 we determined the wind speed of Star A in 2002 and 2009 to be 2210 and $2440 \mathrm{~km} \mathrm{~s}^{-1}$, respectively. This trend for increasing wind speeds between 1999 and 2009 is one that can be traced back to late 1994, at

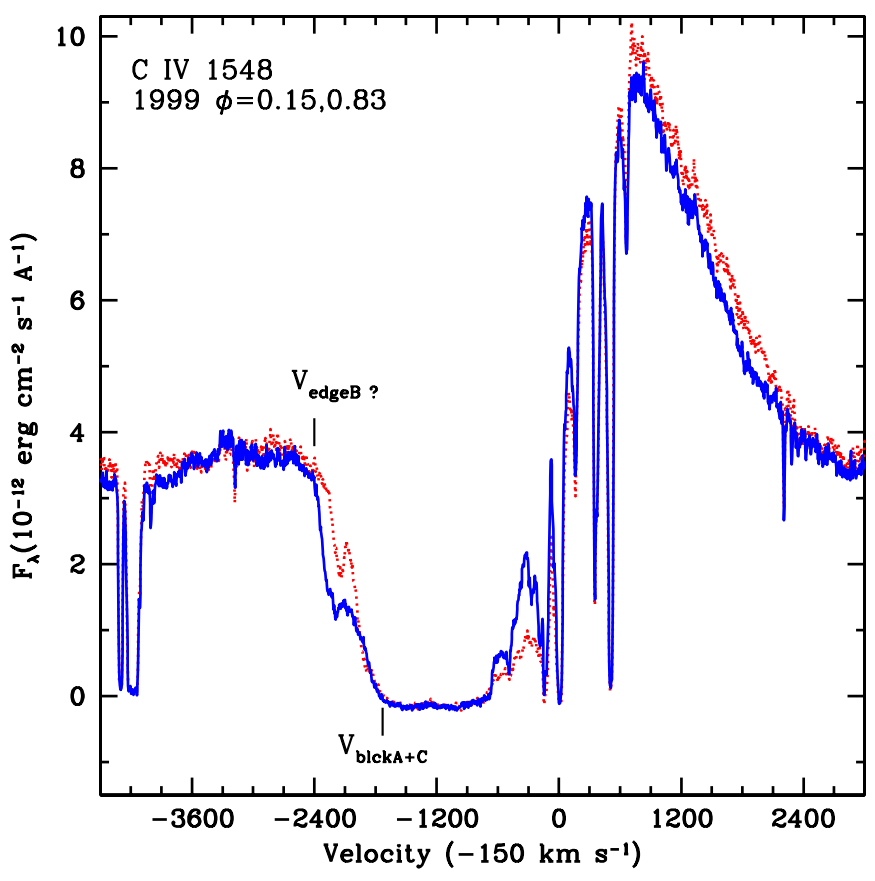

Figure 3. C IV 1548/1550 P Cygni doublet observed in 1999 at orbital phases 0.15 (dotted line) and 0.83 . These phases correspond to the largest projected orbital motion of the stars in the $19.3 \mathrm{~d}$ binary. The "turbulent" edge velocity of $-3100 \mathrm{~km} \mathrm{~s}^{-1}$ seen at $\phi=0.36$ is absent, possibly due to the wind-wind interaction region. The velocity scale is centered on the $1548 \AA$ laboratory wavelength corrected for the SMC motion $\left(+150 \mathrm{~km} \mathrm{~s}^{-1}\right)$.

(A color version of this figure is available in the online journal.)

which time speeds $<500 \mathrm{~km} \mathrm{~s}^{-1}$ were recorded (Koenigsberger et al. 1998a). This is the inverse of HD 5980's behavior in the epochs preceding the eruption; between 1979 and 1991 wind speeds steadily declined.

In the 1979 IUE spectrum obtained at $\phi=0.91$ a plateau extending to $V_{\text {plateau }}=-2670 \mathrm{~km} \mathrm{~s}^{-1}$ is clearly present in the Civ 1550 P Cygni absorption, followed by a gradual rise reaching the continuum level at $-3200 \mathrm{~km} \mathrm{~s}^{-1}$. The other spectra obtained in 1979 also display the plateau which, for example, in the case of the $\phi=0.48$ spectrum extends to $-2750 \mathrm{~km} \mathrm{~s}^{-1}$. 
Table 3

Estimated Wind Speeds

\begin{tabular}{lcccc}
\hline \hline Year & & & & $V_{\text {edge }}($ He II) \\
& Star $A$ & Star $B$ & Star C & Star \\
\hline 1979 & $-2670^{\mathrm{a}}$ & $\ldots$ & -1760 & -2560 \\
1991 & $-2000^{\mathrm{a}}$ & -2320 & -1740 & -1870 \\
1993.7 & $-1610^{\mathrm{b}}$ & $\ldots$ & $\ldots$ & $\ldots$ \\
1994.90 & $-690^{\mathrm{c}}$ & $\ldots$ & $\ldots$ & $\ldots$ \\
1994.94 & $-810^{\mathrm{c}}$ & $\ldots$ & $\ldots$ & $\ldots$ \\
1994.97 & $-860^{\mathrm{c}}$ & $\ldots$ & $\ldots$ & $\ldots$ \\
1994.98 & $-1100^{\mathrm{c}}$ & $\ldots$ & $\ldots$ & $\ldots$ \\
1995.00 & $-1300^{\mathrm{c}}$ & $\ldots$ & $\ldots$ & $\ldots$ \\
1999 & $-1720^{\mathrm{a}}$ & -2300 & -1740 & -1500 \\
2000 & $-2000^{\mathrm{a}}$ & $\ldots$ & -1780 & -1680 \\
2002 & $-2210^{\mathrm{a}}$ & $\ldots$ & -1780 & -2100 \\
2009 & $-2440^{\mathrm{a}}$ & $\ldots$ & -1760 & -2260 \\
\hline
\end{tabular}

Notes.

a From $V_{\text {black }} \mathrm{C}$ IV.

b From P v, FUSE, Koenigsberger et al. 2006.

${ }^{\text {c }}$ From Al III, Koenigsberger et al. 1998a.

This is similar to the $\phi=0.36$ spectrum discussed in Section 3.2. But because none of the 1979-1980 spectra were obtained during eclipse, it is not possible a priori to determine which of the two stars is responsible for the different fast components. However, since the speed determined from spectra obtained in other epochs when Star B eclipses Star A are $\sim-2300 \mathrm{~km} \mathrm{~s}^{-1}$, we are led to conclude that the $\sim-2700 \mathrm{~km} \mathrm{~s}^{-1}$ component in 1979 corresponds to the wind of Star A. A similar analysis leads to the conclusion that by 1991 Star A's wind velocity decreased to $\sim-2200 \mathrm{~km} \mathrm{~s}^{-1}$.

The individual contribution from Star A and Star B may also be identified in other P Cygni lines such as He II 1640 and $\mathrm{Nv} 1240$, lines to which the contribution from Star $C$ is negligible. The $\mathrm{N} v$ line displays a flat portion, analogous to $V_{\text {black }}$, but which does not reach the zero intensity level. Its extent provides the velocity of the slower wind, either that of Star A or Star B, depending on the epoch. A second plateau provides the velocity of the faster wind. The measurements of these two plateaus are listed in Column 6 of Table 1.

The He II 1640 line arises from a transition between two excited states, and thus, the strength of its P Cygni absorption is weaker than that of the $\mathrm{C} I V$ and $\mathrm{N} v$ resonance transitions. Here, the simplest measurement to perform is that of $V_{\text {edge }}$. Because there is no "black" portion in the absorption, it is not clear to what extent "turbulence" contributes to the edge velocity. In addition, some of the spectra show a break in the absorption profile, similar to the plateau seen in the resonance lines, and which may be associated with the second star in the system. The measured values of $V_{\text {edge }}$ and of the plateau (when visible) are listed in Column 5 of Table 1. We find that these values are consistent with the velocity for Star A derived from C IV and $\mathrm{N}$ v. Because the contribution from Star $C$ may be neglected in N v 1240 and He II 1640, we use these lines for the analysis that will be presented in the next section.

The behavior of the three lines is illustrated in Figure 4 for the epochs 2002 and 2009, showing that they all display the wind velocity increase that took place between 2002 and 2009.

A summary of our estimated wind speeds for the three stars at different epochs is given in Table 3. These speeds, as those of Table 1, are corrected for the systematic velocity of the SMC $\left(+150 \mathrm{~km} \mathrm{~s}^{-1}\right)$ but do not include a correction for orbital motion.

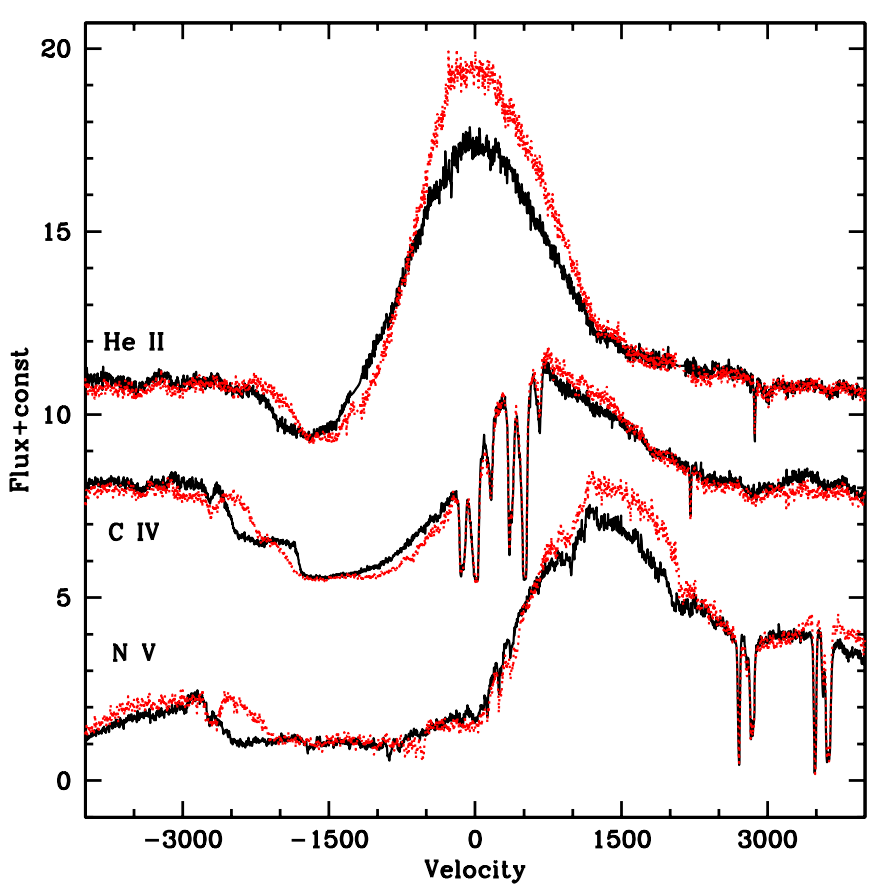

Figure 4. Montage of the $\mathrm{N} \mathrm{v}, \mathrm{C}$ IV, and He II P Cygni profiles in the HST/STIS spectra obtained in 2002 (dots) and 2009. Both spectra correspond to the same orbital phase $\phi=0.99$. The most striking differences are the strength of the emission line and the extent of the absorption component. Note the "step"-like shape of the C IV absorption component in 2009 due to the contribution from Star C $\left(-1760 \mathrm{~km} \mathrm{~s}^{-1}\right)$ and Star A $\left(-2440 \mathrm{~km} \mathrm{~s}^{-1}\right)$. Velocity scale is centered on the laboratory wavelengths of NV1238.821, C IV 1548.187, and He II 1640.47, with a correction of $-150 \mathrm{~km} \mathrm{~s}^{-1}$ to account for the relative SMC motion.

(A color version of this figure is available in the online journal.)

It is important to note that, except for the 1979-1980 epochs, the He II 1640 values of $V_{\text {edge }}$ are systematically smaller than the values derived from $\mathrm{C}$ IV which are listed in Table 3 . It is not clear whether this is caused by the presence of additional emission lines that "fill in" the absorption near its edge ${ }^{13}$ or whether this is a consequence of the excitation structure in the wind.

\section{EMPIRICAL CORRELATIONS}

We have previously shown (Koenigsberger et al. 2010) that the emitted flux in the UV and optical lines increased during the pre-eruption epochs and decreased after $\sim 1999$. Changes in the continuum flux levels have also occurred. Here we show the existence of two correlations and their corollaries.

Line strength versus wind velocity. A decrease in the wind velocity is accompanied by an increase in the strength of the emission lines, i.e., $F_{\text {lines }} \sim V_{\text {wind }}^{-1}$. Figure 5 shows the flux contained in the NIV] 1468 emission line plotted against the P Cygni absorption-line velocity of He II $1640 \AA$ and N v $1240 \AA$. Only data from spectra obtained around $\phi=0.00$ are plotted, so the velocity clearly corresponds to that of Star A.

Continuum intensity versus wind velocity. A decrease in the wind velocity is accompanied by an increase in the strength of the continuum in the UV $(\lambda 1850 \AA)$ and in the visual range, i.e., $F_{\text {contin }} \sim V_{\text {wind }}^{-1}$. This is illustrated in Figure 6 , where the UV continuum flux is plotted against the wind velocity measured from $V_{\text {edge }}$ of He II $1640 \AA$.

These two correlations indicate that changes in the wind velocity are accompanied by changes in the continuum forming 13 The numerous absorptions associated with Star A's active state are absent in
1979-1980 


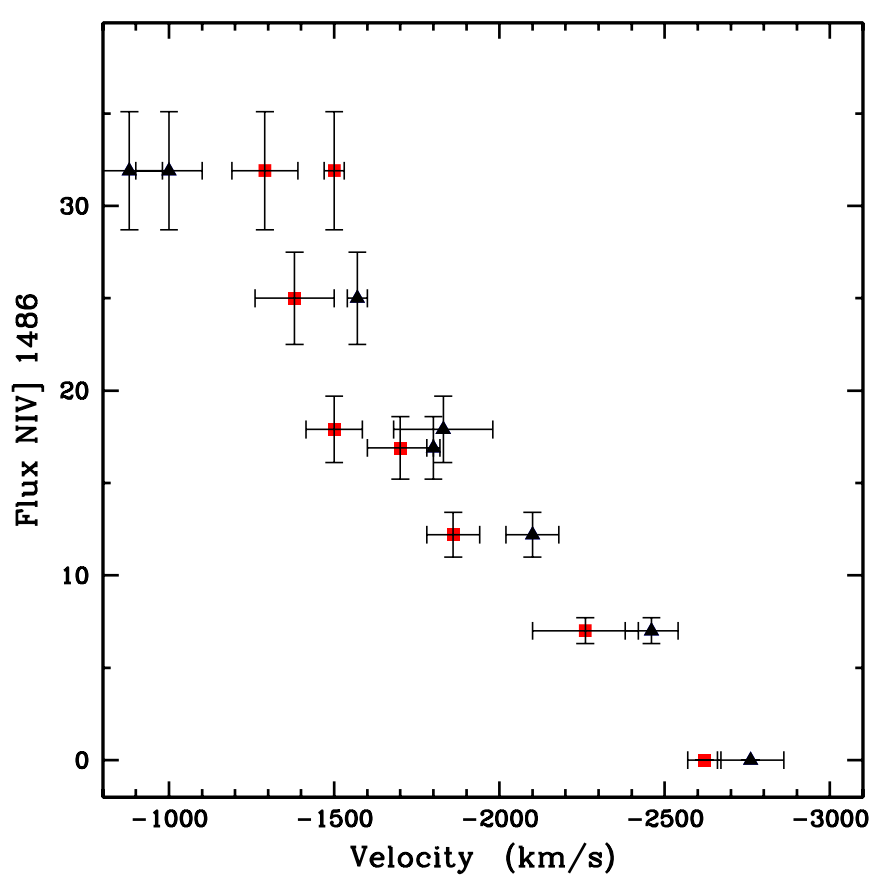

Figure 5. Line strength vs. wind velocity correlation. The NIV] $1486 \AA$ emission-line flux plotted against the wind velocity derived from P Cygni absorption profiles. Only data from spectra obtained around $\phi=0.00$ are plotted. Squares correspond to $V_{\text {edge }}$ of He II $1640 \AA$ and triangles correspond to the saturation portion of $\mathrm{N} \mathrm{v} \mathrm{1238.} \mathrm{Error} \mathrm{bars} \mathrm{in} \mathrm{velocity} \mathrm{correspond} \mathrm{to} \mathrm{estimated}$ uncertainties in each individual measurement while the flux uncertainty is estimated at $10 \%$.

(A color version of this figure is available in the online journal.)

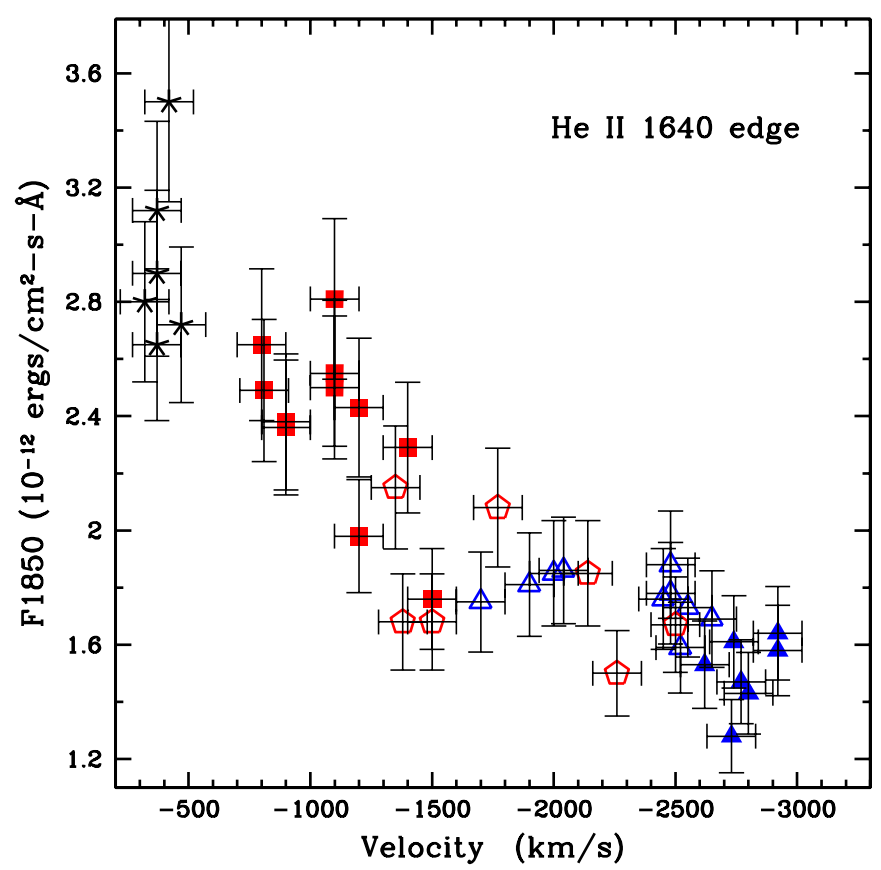

Figure 6. Continuum intensity vs. wind velocity correlation. The UV continuum flux level, $F_{1850}$, plotted against the wind velocity derived from $V_{\text {edge }}$ of He II 1640. Symbols indicate: filled triangles-1979-1981; open squares-1986; open triangles-1989-1991; stars-1994; filled squares-1995; and pentagons-1999-2009. For 1994, we use values of $F_{1850}$ measured on the low dispersion spectra.

(A color version of this figure is available in the online journal.)

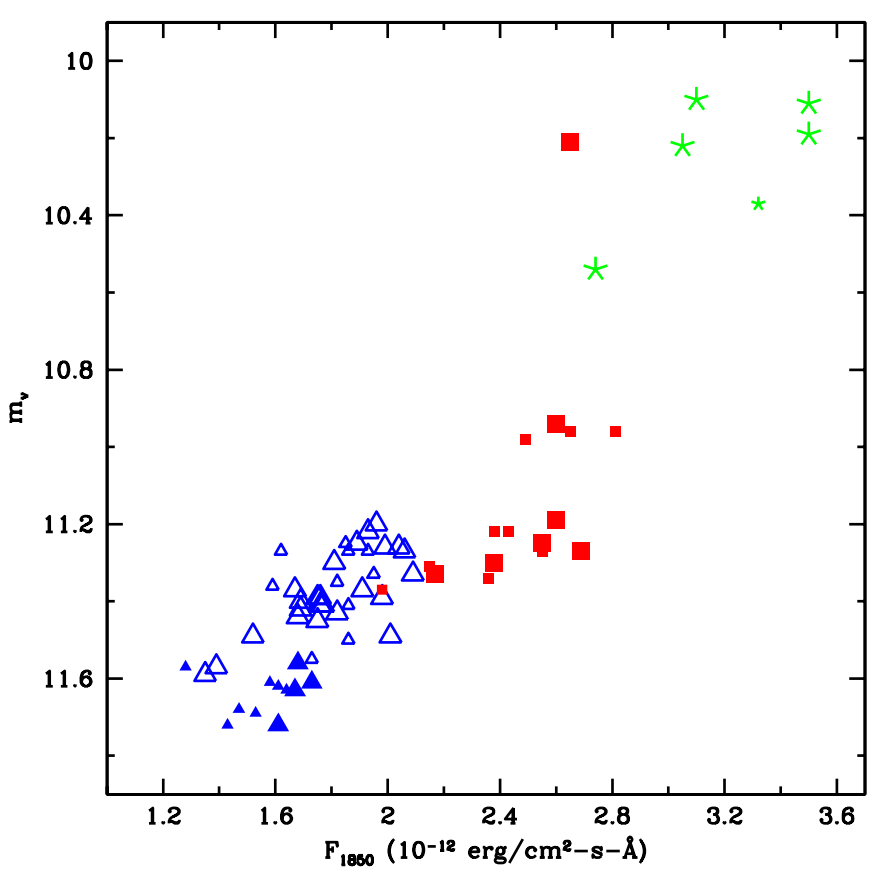

Figure 7. Visual magnitudes derived from the IUE FES counts and the continuum absolute flux values at $1850 \AA$. Symbols indicate: filled triangles-1979-1981; open triangles-1989-1991; stars-1994; filled-1995; Large/small symbols indicate low/high-resolution spectra. The increasing trend in both wavelength regions suggests an increase in bolometric brightness.

(A color version of this figure is available in the online journal.)

region as well as in the more extended line-forming regions. That is, the entire wind structure is affected. It is also important to note that the UV and the visual continuum levels increase or decrease together, as illustrated in Figure 7. Hence, the phenomenon is not simply due to a redistribution in wavelength of the continuum energy.

A corollary of the above relations is that the continuum brightness and emission line strengths increase or decrease together; $F_{\text {cont }} \sim F_{\text {lines }}$. This is illustrated in Figure 8, where the strengths of N IV] 1486 and N III 1750 are plotted against the flux in the continuum at $1850 \AA$. The different epochs of observation lie in different locations within this diagram. These results suggest that the physical phenomenon causing the changes in Star A involves an overall increase or decrease in the energy that is emitted, i.e., bolometric luminosity variations.

Let us momentarily assume that Star A's continuum emits as a blackbody. The ratio $F_{\text {vis }} / F_{1850}=10^{-0.4 * m(\text { FES })} / F_{1850}$ at constant luminosity is an almost linear function of the flux at $1850 \AA$ with $F_{\text {vis }} / F_{1850}$ decreasing with the increasing temperature (continuous line in Figure 9). The observed points in Figure 9 are clearly separated in two groups. The points before the eruption and after 1995 lie along a curve similar to the one described by the blackbody, while the points obtained during the eruption are displaced from this correlation. The temperature decreased during the eruption but contrary to what might be expected, the absolute flux at $1850 \AA$ increased, as seen in the observed $F_{\text {vis }} / F_{1850}$ correlation. To account for this, the luminosity must have increased by a factor of $\sim 6$ (dashed line in Figure 9). This leads to the conclusion that the 1994 eruption involved a luminosity increase, a conclusion that is strengthened by the results of the CMFGEN modeling described in the next sections. The dispersion of the points around the blackbody curve during the other epochs points to some further changes in the luminosity although at a much smaller scale. 

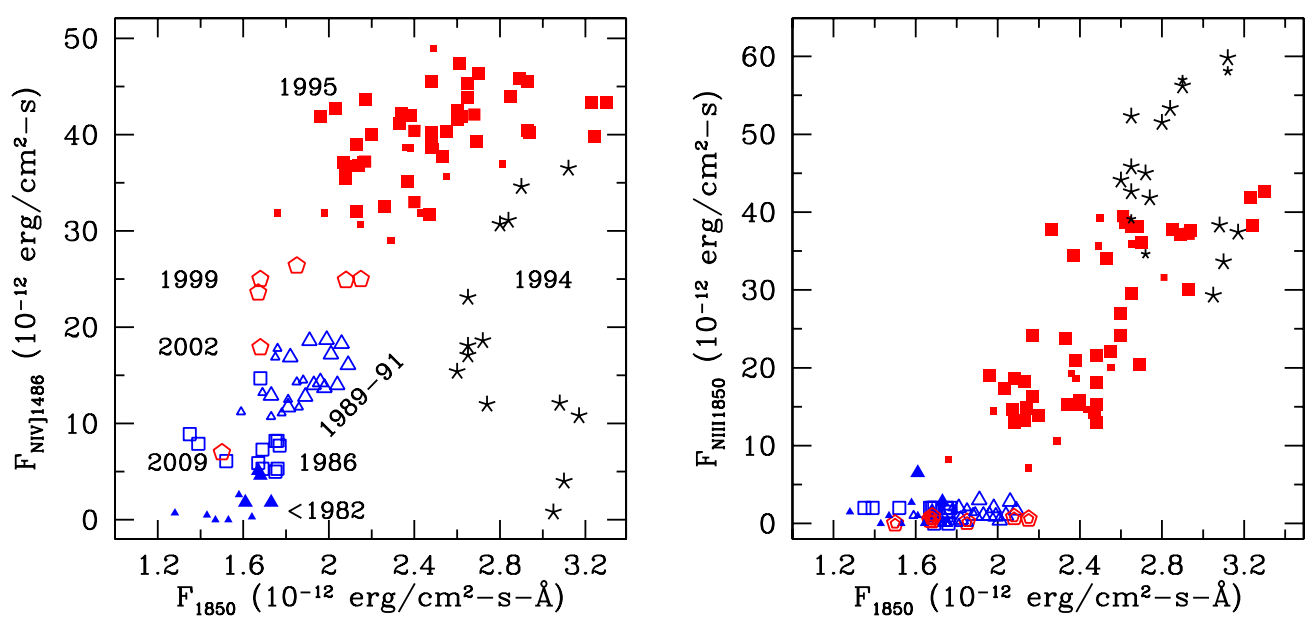

Figure 8. Absolute flux contained in the N IV]1486 ̊ (left) and N III 1750+1754 A (right) emission lines plotted as a function of the absolute continuum flux at $1850 \AA ̊$ A The different epochs are indicated by different symbols: filled triangles—1979-1981; open squares—1986; open triangles—1989-1991; stars—1994; filled squares-1995; and pentagons-1999-2009. Large symbols correspond to values obtained from the IUE low-resolution spectra and HST/STIS data; small symbols to high-resolution $I U E$ spectra. Uncertainties are $\sim 10 \%$ of the flux values.

(A color version of this figure is available in the online journal.)

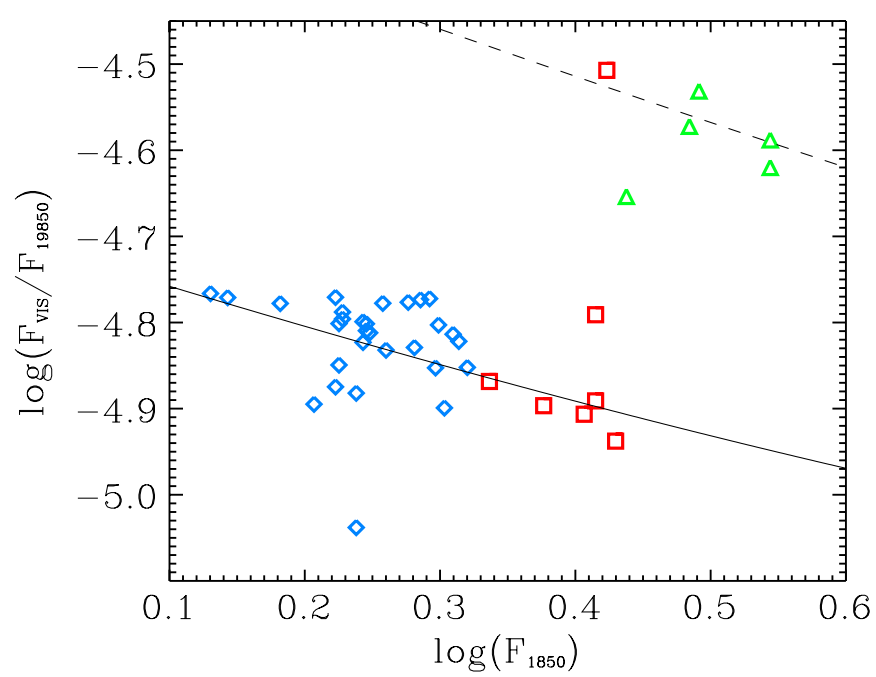

Figure 9. Ratio of the visual flux, $F_{\mathrm{VIS}}$ converted from the IUE FES magnitudes and $F_{1850}$ from Table 2, plotted against $F_{1850}$. Small open squares-data before 1994; triangles — data of 1994; large squares - data after 1995 January. The continuous line corresponds to the relation of $F_{\mathrm{VIS}} / F_{1850}$ vs. $F_{1850}$ for a blackbody energy distribution flux of different temperatures assuming a constant luminosity. The dashed line is the same relation but with a luminosity that is six times larger, implying that the 1994 eruption involved a significant change in the luminosity.

(A color version of this figure is available in the online journal.)

The interpretation of the line strength versus wind velocity correlation is not straightforward. The larger $V_{\infty}$ increases the transformed radius,

$$
R_{t}=R_{\text {star }}\left(\frac{V_{\infty} / 2500 \mathrm{~km} \mathrm{~s}^{-1}}{\dot{M} / 10^{-4} M_{\odot} \mathrm{yr}^{-1}}\right)^{\frac{2}{3}}
$$

(Schmutz et al. 1989) reducing the line strength. Thus, having in mind that $V_{\infty}$ has been changing, one cannot directly interpret the weaker lines as a result of a lower mass-loss rate. To proceed further in the interpretation of the line intensity variations it is necessary to model the spectra of Star A, which will be done in the next section.

\section{CMFGEN MODELS OF STAR A AND STAR C}

As previously described, Star A undergoes two modes of large-scale variability: (1) a long term ( $\sim 40$ years $) S$ Dortype variability and (2) an eruptive mode, as occurred in 1993-1994, shortly before the maximum of the S Dor cycle. The observed changes in lines and continuum fluxes that were described in Section 4 point to two separate physical states of the wind corresponding to these two modes. In order to gain further insight into the processes which drive the variability, we modeled the spectra obtained in 1994, 2000, 2002, and 2009 which are described in Section 2. These spectra correspond to times when the emission from Star A dominates over Star B's emission. However, Star $C$ is always present in the observations, so a model for this star was also constructed in order to adequately compare the models with the data. All comparisons are made against the sum of the fluxes from the Star A and Star $C$ models.

We modeled the spectrum of Star A using a new version of CMFGEN code. In this version the spectrum forming region of the star is modeled as a hydrostatic photosphere and a wind attached to it. The wind is described with the usual $\beta$ law. The photosphere is specified with its gravity $(\log g)$ and temperature $T_{10}$ at the Rosseland optical depth $\tau_{\text {Ross }}=10$. This large value of $\tau_{\text {Ross }}$ is chosen so that models with different mass-loss rates are comparable. The density of the photosphere is calculated to satisfy the equation:

$$
\nabla P=-\rho\left(g_{\text {grav }}-g_{\text {rad }}\right),
$$

where $g_{\text {grav }}$ is the specified gravitational acceleration and $g_{\text {rad }}$ is the calculated acceleration due to the radiation pressure. The solution defines the density as a function of the radius. This defined density and the adopted $\dot{M}$ together with the equation of continuity defines the velocity as a function of the radius. The velocity increases with decreasing density and it is connected to the wind velocity at a prescribed point which we choose to be $10 \mathrm{~km} \mathrm{~s}^{-1}$. Once the velocity and density structures are specified, the radiation transport equation is solved consistently with the equations of statistical equilibrium and the energy balance as described in Hillier \& Miller (1998). Several iterations of this procedure are performed until the density structure is consistent 

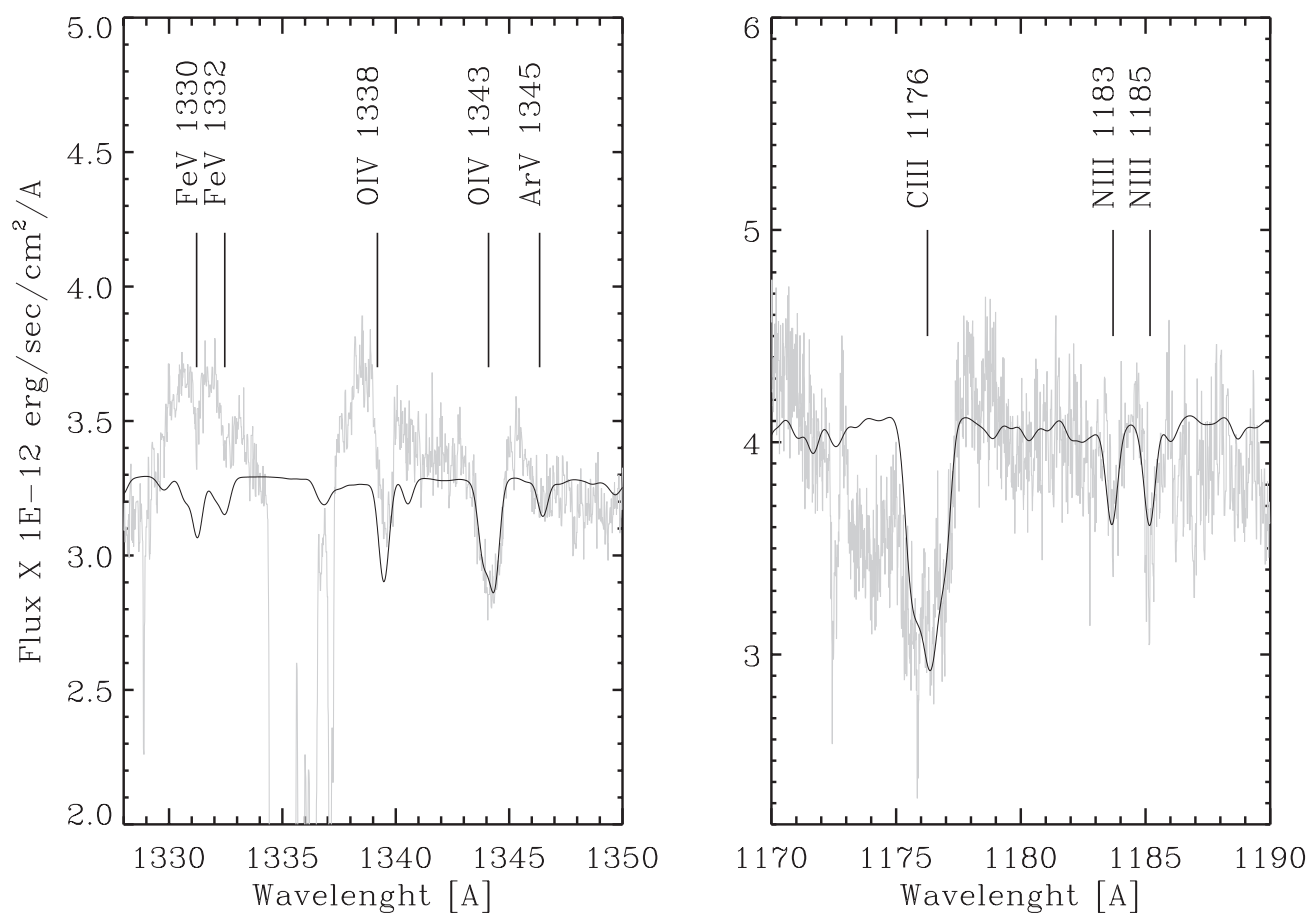

Figure 10. Fragments of 2002 HST/STIS and FUSE spectra (gray line) compared with the Star C model (black line). The model spectrum is shifted to the level of the observed spectrum which is a sum of Star A and Star C fluxes.

with the radiation force and the temperature distribution. The reference point at $\tau_{\text {Ross }}=10$ is usually situated at $\mathrm{V}(\mathrm{r}) \sim$ $5 \mathrm{~km} \mathrm{~s}^{-1}$, below the connection point so the model parameters $T_{10}$ and $\log g$ apply to the underlying photosphere rather than the wind itself.

The model of the star is specified by several parameters which can be combined into two groups. In the first group, there is the chemical composition, the atomic data and the stellar mass. For the analysis of HD 5980, the values of these parameters are fixed for all epochs. The second group includes the stellar luminosity, the gravity acceleration, the temperature, the terminal velocity and the mass-loss rate. For Star $C$, only one model fit was performed and we assume that all its derived parameters remain constant over time. For Star A, model fits were performed for each of the four spectra described in Section 2.

\subsection{Star C Model}

Numerous photospheric absorption lines belonging to Star $C$ (Koenigsberger et al. 2002) are clearly present in the 2009 spectrum. They were used to construct a model for this star. Hunter et al. (2007) found that $\mathrm{Si}$ and $\mathrm{Mg}$ composition in B stars in NGC 346 is $\sim 0.2$ of the solar abundance (Table 6). Assuming this is representative for all metals heavier than $\mathrm{O}$, we adopted that chemical composition. The temperature of the model was fixed by the O IV 1339-43 $\AA$ and O III $5592 \AA$ lines. The model parameters were adjusted to attain a reasonable fit to the depth of the He I and H I optical absorption lines, clearly visible on top of the emission lines. The wind velocity was set to the observed value of $V_{\text {black }}=1770 \mathrm{~km} \mathrm{~s}^{-1}$ and the massloss rate was restricted to the minimal value for which the $\mathrm{C}$ IV 1548-50 doublet is saturated. The luminosity of Star $C$ was fixed so that its continuum coincide with the "step" of the observed C IV 1548/50 doublet. We adopt the CNO abundances of Sk 80 (Crowther et al. 2002), with which the lines of O III $5592 \AA$, C III $1175 \AA$ and C III $2297 \AA$ and N III 1183-85 A (Figure 10) are adequately reproduced. The adopted parameters are shown in Column 6 of Table 6 and the spectrum is shown in Figure 11. The model derived for Star $C$ does not have significant emission lines in the UV-optical region other than C IV 1548-50 , N IV $1718 \AA$, and N v 1239-43 A. The comparison between the observed absorption lines and the computed spectrum suggests a rotational velocity $V_{\text {rot }} \sin i=80 \pm 15 \mathrm{~km} \mathrm{~s}^{-1}$ and a radial velocity $V_{r} \sim 60 \mathrm{~km} \mathrm{~s}^{-1}$ with respect to the Star $A+\operatorname{Star} B$ center of mass.

It is important to note that Schweickhardt (2000) found periodic radial velocity variations in the photospheric absorption associated with Star $C$, with $\mathrm{P}_{\mathrm{StarC}} \sim 96.5$ days, and suggested that it is itself a binary system. Foellmi et al. (2008) also found RV variations in the O III 5592.4 line consistent with Schweickhardt's (2000) conclusion. It is thus important to keep in mind that our model for Star $C$ corresponds to the combined spectra of two objects. A second point to keep in mind is that it remains to be determined whether this binary is gravitationally bound to the Star $A+\operatorname{Star} B$ pair or whether it is merely a line-of-sight projection.

\subsection{Star A}

In this section we describe the procedure for computing the model spectrum for Star A. Because Star $C$ is in view at all times, the computed spectrum of Star $C$ described above was added to all models of Star $A$ before comparison with the observed spectrum. In the cases when only the normalized observed spectrum is available we compared the observations with a model flux calculated as

$$
\begin{gathered}
F(\text { model })=\frac{\left(F_{\lambda}(\operatorname{Star} \mathrm{A})+F_{\lambda}(\operatorname{Star} \mathrm{C})\right)}{\left(F_{\text {cont }}(\operatorname{Star} \mathrm{A})+F_{\text {cont }}(\operatorname{Star} \mathrm{C})\right)} . \\
\text { 5.2.1. Chemical Composition }
\end{gathered}
$$

We constructed a fairly complex model including most of the important atoms in several ionization stages (Table 4). 

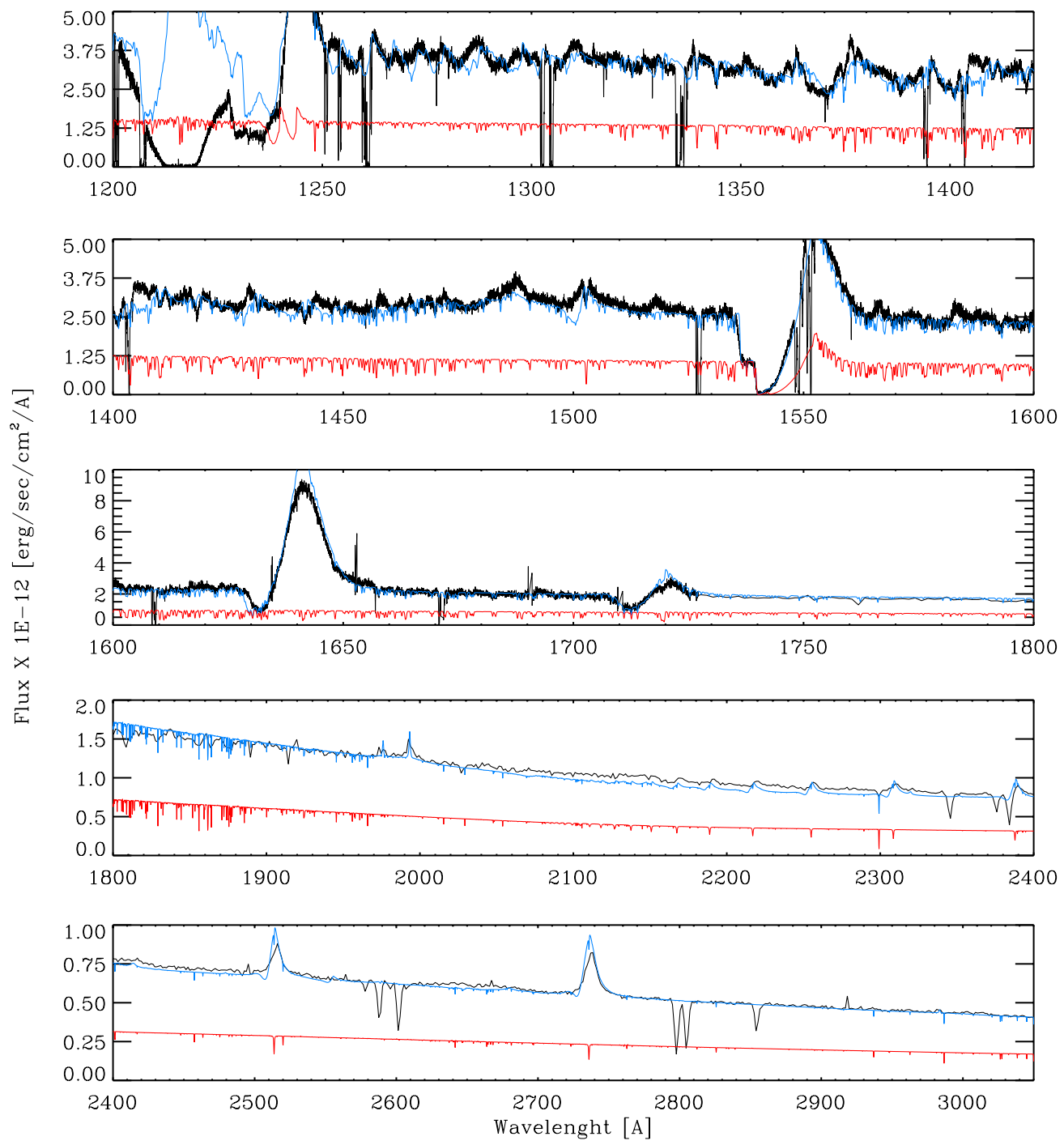

Figure 11. Comparison between the HST/STIS spectrum obtained in 2009 September (black) and the sum of the current best model for Star A at this epoch and Star $C$ model. The red line represents the computed spectrum of Star $C$ alone. Note the coincidence of the Star C's continuum and the step in the blue wind of the C Iv $1548 / 50$ Å P Cyg absorption.

Table 4

Ions and Number of Levels Treated in the Model

\begin{tabular}{|c|c|c|c|c|c|c|c|c|c|}
\hline Elements & I & II & III & IV & V & VI & VII & VIII & IX \\
\hline $\mathrm{H}$ & $20 / 30$ & & & & & & & & \\
\hline $\mathrm{He}$ & $27 / 39$ & $13 / 30$ & & & & & & & \\
\hline $\mathrm{C}$ & & $40 / 92$ & $51 / 84$ & $59 / 64$ & & & & & \\
\hline $\mathrm{N}$ & & $45 / 85$ & $41 / 82$ & $44 / 76$ & $41 / 49$ & & & & \\
\hline $\mathrm{O}$ & & $54 / 123$ & $88 / 170$ & $38 / 78$ & $32 / 56$ & $25 / 31$ & & & \\
\hline $\mathrm{Ne}$ & & $42 / 242$ & $40 / 182$ & $53 / 355$ & $37 / 166$ & $36 / 202$ & & & \\
\hline $\mathrm{Al}$ & & & $21 / 65$ & & & & & & \\
\hline $\mathrm{Si}$ & & & $20 / 34$ & $22 / 33$ & & & & & \\
\hline $\mathrm{P}$ & & & & $36 / 178$ & $12 / 62$ & & & & \\
\hline S & & & $13 / 28$ & $51 / 142$ & $31 / 98$ & $28 / 58$ & & & \\
\hline $\mathrm{Cl}$ & & & & $40 / 129$ & $26 / 80$ & $18 / 44$ & $17 / 28$ & & \\
\hline $\mathrm{Ar}$ & & & $10 / 36$ & $31 / 105$ & $38 / 99$ & & & & \\
\hline $\mathrm{Ca}$ & & & & $43 / 378$ & $73 / 613$ & $47 / 108$ & $48 / 288$ & $45 / 296$ & $39 / 162$ \\
\hline $\mathrm{Cr}$ & & & $30 / 145$ & $29 / 234$ & $30 / 223$ & $30 / 215$ & & & \\
\hline $\mathrm{Mn}$ & & & & $39 / 464$ & $16 / 80$ & $23 / 181$ & $20 / 203$ & & \\
\hline $\mathrm{Fe}$ & & & $104 / 1433$ & $100 / 1000$ & $139 / 1000$ & $44 / 433$ & $29 / 153$ & & \\
\hline $\mathrm{Ni}$ & & & & $115 / 1000$ & $152 / 1000$ & $62 / 1000$ & $37 / 308$ & $34 / 325$ & $34 / 363$ \\
\hline
\end{tabular}


The main properties of the wind were obtained for each epoch. If the observed spectrum at one epoch required an adjustment of the chemical composition, the change was made in all four epochs and the models were recalculated and the consistency checked.

The He composition was determined by the decrement of the $\mathrm{H} \mathrm{I}$ and $\mathrm{H} \mathrm{I}+\mathrm{He}$ II optical lines. We fitted the lines in the spectrum obtained in 1994 and found that $\mathrm{N}(\mathrm{He}) / \mathrm{N}(\mathrm{H})=1.0 \pm 0.2$ by number. This value was fixed for all four spectra. Due to the significant abundance of $\mathrm{He}$, the mean atomic mass of the gas is larger than the Solar. In order to be able compare Star A with other objects having different $\mathrm{He} / \mathrm{H}$ ratios we present the composition of all other elements by their mass fractions.

The carbon abundance was constrained mainly by C IV 5804/12 $\AA$ and C III $1178 \AA$ as observed in the 2002 FUSE spectrum. The strong C IV 1548-50 doublet is not sensitive to the abundance. The N IV $7123 \AA$ line was used as a nitrogen abundance indicator, and consistency was checked using the UV N III, N IV, and N v lines. Several iterations on all models at all epochs were made until a consistent abundance of nitrogen was obtained.

We do not observe any strong oxygen lines in the spectrum. The O IV 1338-41 $\AA$ observed in STIS spectra are formed in Star C's wind. The weakness of these lines in Star A's spectrum sets an upper limit to the $\mathrm{O}$ composition to 0.1 of the $\mathrm{SMC}$ value (1/50 of the solar composition). The phosphorus abundance was set to 0.05 solar. The predicted $\mathrm{PV} 1118-28 \AA$ lines based on this value agree with the 2002 observations. S v $1502 \AA$ line was fit with a sulfur abundance equal to 0.05 solar. The same abundance fits reasonably well S vi $933 \AA$ as well. Aluminum abundance of 0.1 solar fits Al III 1855/63 Å doublet observed in the 1994 spectrum.

Finally, we adopted $\mathrm{Fe} / \mathrm{H}=0.1(\mathrm{Fe} / \mathrm{H})_{\odot}$ by mass since this abundance reproduces well the $\mathrm{Fe} \mathrm{V}$ and $\mathrm{Fe}$ IV lines in the spectra of the 2000, 2002, and 2009 epochs. This iron abundance is lower than the one adopted for $\operatorname{Star} C\left(\mathrm{Fe} / \mathrm{H}=0.2(\mathrm{Fe} / \mathrm{H})_{\odot}\right)$ but we did not perform a rigorous analysis of the abundances of Star $C$ and we cannot exclude that it has the same $\mathrm{Fe} / \mathrm{H}$ abundance $\sim 0.1(\mathrm{Fe} / \mathrm{H})_{\odot}$ as of Star A. Nevertheless we kept the $\mathrm{Fe} / \mathrm{H}$ abundance of Star $C$ to the commonly accepted value. The lower iron abundance of Star $A$ is consistent with the result derived from a comparison of the wind-eclipse effects in HD 5980 and the Galactic WR system HD 90657 (Koenigsberger et al. 1987). ${ }^{14}$ For the elements $\mathrm{Ne}, \mathrm{Ar}, \mathrm{Cl}, \mathrm{Ca}, \mathrm{Cr}, \mathrm{Mg}$, and $\mathrm{Ni}$, we adopted a 0.1 times Solar composition similar to the iron abundance. The spectrum does not show any observable spectral features arising from these species but they are important for the line blanketing and the radiation force and were included in the model.

The composition of all other elements included in the model was also set to 0.10 of the solar value. The final chemical composition adopted for the four epochs is shown in Table 5. Note that the overall abundance for Star A is 0.1 Solar while that which was used for the Star $C$ model is 0.2 Solar. The uncertainties in the model fits lead to uncertainties in the chemical compositions of \pm 0.1 dex, so that these two metallicity values are consistent, within the uncertainties.

\subsubsection{Wind Velocity}

The calculated spectrum of a model is sensitive to several parameters. The first two parameters are the wind velocity law

\footnotetext{
14 This result refers to the Fe abundance in Star B's wind, derived from a comparison between the Fe v pseudocontinuum and the N IV $1718 \AA$ line.
}

Table 5

Chemical Composition of Star A Compared to Other Objects

\begin{tabular}{lrrrcrr}
\hline \hline Element & Star A $^{\mathrm{a}}$ & Star $^{\mathrm{a}}$ & SMC $^{\mathrm{b}}$ & NGC346 $^{\mathrm{c}}$ & AG Car $^{\mathrm{d}}$ & Sun $^{\mathrm{e}}$ \\
\hline $\mathrm{H}$ & 11.304 & 11.853 & $\ldots$ & $\ldots$ & 11.868 & 11.868 \\
$\mathrm{He}$ & 11.902 & 11.455 & $\ldots$ & 11.381 & 12.097 & 11.397 \\
$\mathrm{C}$ & 7.484 & 8.233 & $\ldots$ & $\ldots$ & 8.694 & 9.334 \\
$\mathrm{~N}$ & 9.000 & 7.999 & 7.526 & 7.521 & 10.161 & 8.791 \\
$\mathrm{O}$ & 6.176 & 9.057 & 9.224 & 9.219 & 8.679 & 9.729 \\
$\mathrm{Ne}$ & 8.241 & 8.542 & 8.474 & 8.470 & $\ldots$ & 9.010 \\
$\mathrm{Na}$ & 6.538 & 6.839 & $\ldots$ & $\ldots$ & $\ldots$ & 7.396 \\
$\mathrm{Mg}$ & 7.811 & 8.111 & $\ldots$ & $\ldots$ & $\ldots$ & 8.780 \\
$\mathrm{Al}$ & 6.747 & 7.049 & $\ldots$ & $\ldots$ & 8.056 & 7.666 \\
$\mathrm{Si}$ & 7.844 & 8.146 & $\ldots$ & $\ldots$ & 9.123 & 8.823 \\
$\mathrm{P}$ & 5.787 & 6.086 & $\ldots$ & $\ldots$ & $\ldots$ & 6.716 \\
$\mathrm{~S}$ & 7.562 & 7.863 & 7.966 & 7.961 & $\ldots$ & 8.511 \\
$\mathrm{Cl}$ & 5.896 & 6.196 & $\ldots$ & $\ldots$ & $\ldots$ & 6.914 \\
$\mathrm{Ar}$ & 6.009 & 7.310 & 7.291 & 7.286 & $\ldots$ & 7.646 \\
$\mathrm{Ca}$ & 6.809 & 5.865 & $\ldots$ & $\ldots$ & $\ldots$ & 7.778 \\
$\mathrm{Ti}$ & 5.516 & 7.090 & $\ldots$ & $\ldots$ & $\ldots$ & 6.445 \\
$\mathrm{Cr}$ & 6.230 & 6.531 & $\ldots$ & $\ldots$ & $\ldots$ & 7.221 \\
$\mathrm{Mn}$ & 5.975 & 6.274 & $\ldots$ & $\ldots$ & $\ldots$ & 6.995 \\
$\mathrm{Fe}$ & 8.134 & 8.436 & $\ldots$ & $\ldots$ & 9.442 & 9.062 \\
$\mathrm{Ni}$ & 6.865 & 7.164 & $\ldots$ & $\ldots$ & $\ldots$ & 7.863 \\
\hline & & & $\ldots$ & & &
\end{tabular}

Notes.

a This paper.

b Hunter et al. 2007.

c Peimbert et al. 2000.

d Groh et al. 2009.

e Grevesse et al. 2007.

and the terminal speed, $V_{\infty}$. The Fe $\mathrm{V}$ and Fe VI lines in the 1270-1450 A region are optically thinner than the stronger lines present in the spectrum, and thus they are more sensitive to properties of the inner wind regions, particularly the velocity law. We find that a $\beta \geqslant 2$ velocity law adequately reproduces their line-profiles. Values of $\beta<2$ produce profiles with a more "box-like" shape than observed. Thus, we set $\beta=2$. The value of $V_{\infty}$ was initially chosen for each epoch from Table 3. However, we found it challenging to achieve a good fit to the $\mathrm{P}$ Cygni absorption components of all lines with the same value of $V_{\infty}$. In general, the value of $V_{\infty}$ deduced from the $\mathrm{C}$ IV line leads to a model in which the extent of the He II $1640 \AA$ and P V 1118-28 $\AA$ P Cygni absorption is greater than observed (Figure 12). The difference is $\sim 500 \mathrm{~km} \mathrm{~s}^{-1}$. As mentioned in Section 2, the wind velocity determined from the He II 1640 $\mathrm{P}$ Cygni edge is systematically smaller than that derived from $\mathrm{C}$ IV (listed in Table 3 ). A slower wind velocity law (i.e., $\beta>2$ ) reduces this discrepancy. However, in the case of N v $1240 \AA$ A our models require a faster $V_{\infty}$ than that derived from $\mathrm{C}$ IV, although the measurements of Table 2 give values that are consistent, within the uncertainties, with C IV. Increasing the value of $\beta$ does not eliminate this inconsistency in the model. Similar discrepancies are observed in some central stars of planetary nebulae (Morisset \& Georgiev 2009; Herald \& Bianchi 2011; A. Arrieta et al. 2011, in preparation). This phenomenon needs further investigation. For the purposes of this paper we used the $V_{\infty}$ as derived from the $\mathrm{C}$ IV 1548-50 $\AA$ line and $\beta=2$.

\subsubsection{Stellar Mass and Luminosity}

No feature in the spectrum is clearly dependent on the gravity acceleration. This is not surprising given that the continuum forming region extends beyond the photosphere. But the mass of the star, $M \sin i, i$ being the orbital inclination, has been 

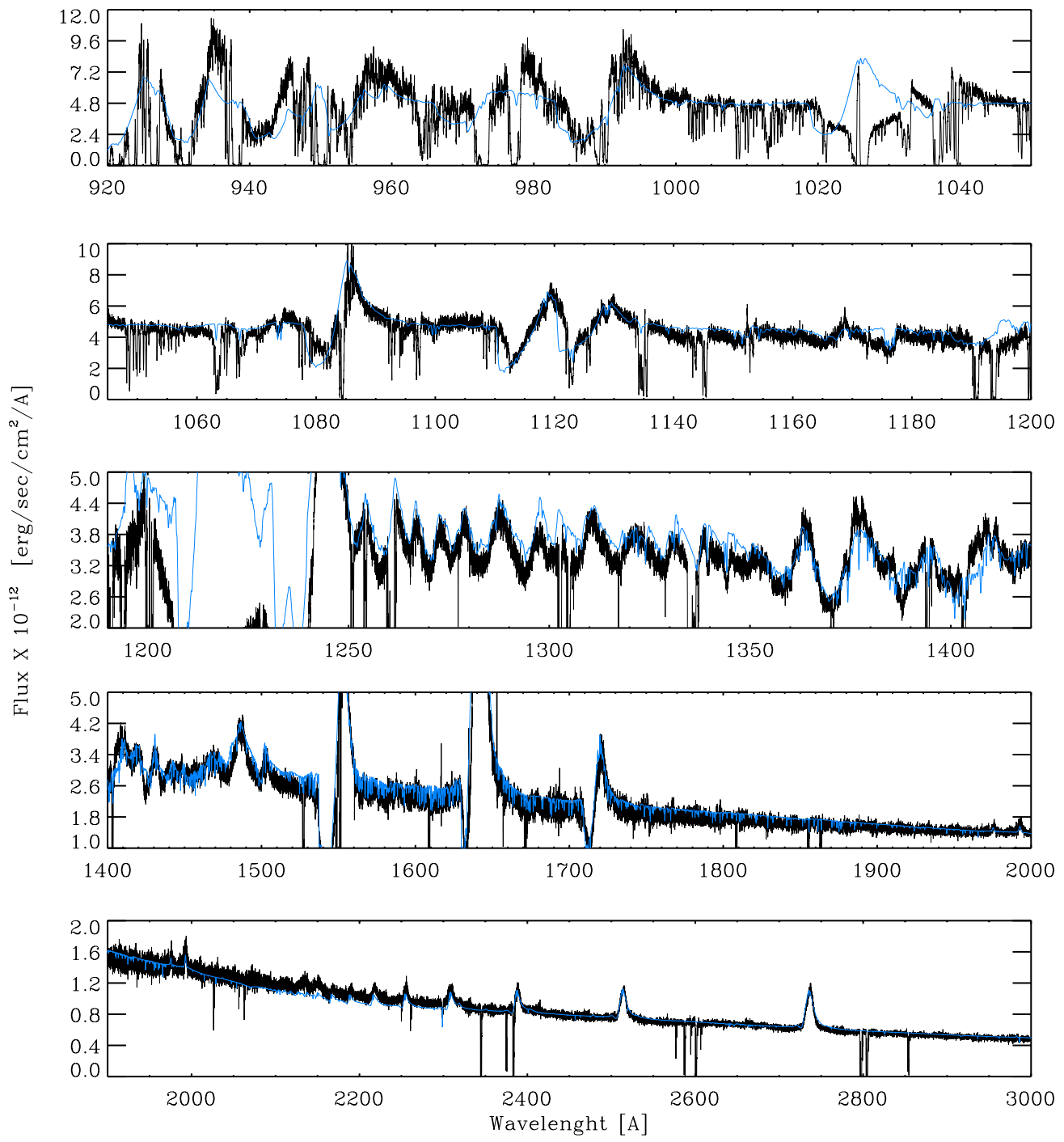

Figure 12. As in Figure 13 but for the 2002 April epoch.

estimated from the analysis of the light curve and radial velocity curve to be between 60 and $80 M_{\odot}$. Our modeling shows that at the observed luminosities and mass-loss rates, a star with a mass smaller than $90 M_{\odot}$ is above the Eddington limit. To keep the star stable, we assume a mass of $90 M_{\odot}$ which, within the errors, is consistent with the orbital solution and keeps the stellar photosphere below the Eddington limit. The $\log g$ was adjusted so the mass of the star is maintained the same for all epochs.

The luminosity at different epochs was determined from the continuum level of the flux calibrated UV spectra. We assume a distance to the SMC of $64 \mathrm{kpc}$ which is an average of the values obtained by Hilditch et al. (2005) and North et al. (2010). The reddening was determined by comparing the model and observed spectral energy distribution in the $920 \AA-11500 \AA$ range and using the Cardelli et al. (1989) reddening law. We obtained a good fit with $E(B-V)=0.065 \pm 0.005$ and $R=A_{V} / E(B-V)=3.1$ which was used for all epochs.

\subsubsection{Temperature, Mass-loss Rate, and Clumping}

The temperature and the mass-loss rate are the most difficult parameters to determine. The change in the spectrum over the different epochs was so great that one cannot use the same diagnostic feature for the analysis for all epochs. The ever present helium lines are affected by Star $B$ in a yet unclear way so we avoided using them. We concentrated on lines which significantly change from epoch to epoch and therefore can safely be assumed to arise in Star A. Nitrogen is present as N III, $\mathrm{N}$ IV, and $\mathrm{Nv}$ in different epochs and due to this variability we deduce that Nitrogen lines are formed mostly in Star A's wind. We used the N IV 1486] line as a mass-loss diagnostic and the N III $1750 \AA$ and $4640 \AA$ lines (when present) were used to constrain the temperature. Although we did not use the helium lines for this analysis, the fits to the He II lines are reasonably good in most of the epochs. He I lines are reasonably fit in 1994 and 2000 epochs but the models underestimate them in 2009. The profile of He I lines is also narrower for the observed $V_{\infty}$. This suggests that at least part of the observed He I $5876 \AA$ emission originates in wind-wind interaction region.

It is now well established that the winds of the massive stars are not homogeneous but rather made of clumps, although the true nature of the clumping is not known. The main effect of the clumping in the WR winds is the absence of electron scattering wings of the strong emission lines. Following the same procedure as in Hillier et al. (2003) we used the volume filling factor $f$ prescription. We adjusted $f$ until we fit the red wing of He II $4686 \AA$ line and then check the consistency with the 
Table 6

Model Fit Results

\begin{tabular}{|c|c|c|c|c|c|c|c|}
\hline Parameter & $1994 \mathrm{Sept}^{\mathrm{a}}$ & $1994 \mathrm{Dec}^{\mathrm{b}}$ & $1994 \mathrm{Dec}^{\mathrm{c}}$ & $2000^{c}$ & $2002^{c}$ & $2009^{c}$ & Star $C^{\mathrm{c}}$ \\
\hline JD - 240000.0 & $\ldots$ & 49716.6 & 49716.6 & 51655.1 & 52386.6 & 55083.9 & $\ldots$ \\
\hline$V$ (mag) (system) & $\ldots$ & 11.12 & $11.12^{\mathrm{b}}$ & 11.3 & 11.6 & $\ldots$ & $\ldots$ \\
\hline$R_{10}\left(R_{\odot}\right)$ & $\ldots$ & $48^{\mathrm{d}}$ & 28 & 20.4 & 21 & 19.3 & 23.5 \\
\hline$R_{s}\left(R_{\odot}\right)$ & $\ldots$ & $\ldots$ & 21.5 & 24.3 & 21.2 & 19.6 & 25.0 \\
\hline$R_{2 / 3}\left(R_{\odot}\right)$ & 280 & $\ldots$ & 124 & 34 & 32 & 28 & 24.2 \\
\hline$\tau_{s}$ & $\ldots$ & $\ldots$ & 25.8 & 4.7 & 3.7 & 1.8 & 0.005 \\
\hline$T_{\text {eff }}(\mathrm{kK})$ & $\ldots$ & $\ldots$ & 23 & 37.3 & 40 & 43 & 32 \\
\hline$T_{*}(\mathrm{kK})$ & 23 & $35.5^{\mathrm{d}}$ & 47 & 48 & 50 & 47 & 33 \\
\hline$T_{s}(\mathrm{kK})$ & $\ldots$ & $\ldots$ & 100 & 57 & 58 & 60 & 27 \\
\hline$\dot{M} / \sqrt{f}\left(10^{-5} M_{\odot} \mathrm{yr}^{-1}\right)$ & 80 & 100 & 111 & 35 & 25 & 23 & 0.06 \\
\hline $\log \left(L / L_{\odot}\right)$ & 7.05 & 6.48 & 6.57 & 6.30 & 6.39 & 6.39 & 5.77 \\
\hline$V_{\infty}\left(\mathrm{km} \mathrm{s}^{-1}\right)$ & 500 & 600 & 750 & 2000 & 2200 & 2440 & 1800 \\
\hline$V_{\mathrm{esc}}\left(\mathrm{km} \mathrm{s}^{-1}\right)$ & 464 & $\ldots$ & 642 & 832 & 895 & 932 & $\ldots$ \\
\hline$\Gamma$ & $0.75^{\mathrm{e}}$ & $\ldots$ & 0.75 & 0.53 & 0.53 & 0.53 & $\ldots$ \\
\hline
\end{tabular}

Notes.

a Drissen et al. 2001.

b Koenigsberger et al. 1998b.

c This paper.

d "Core" radius, $R_{*}$, and corresponding temperature; in Koenigsberger et al. (1998b), $R_{*}$ is the inner boundary of the model atmosphere, where the expansion velocity is negligible.

e Adopted value same as in 1994 model.

other strong H I + He II lines. The observed electron scattering wings are very weak which restrict the volume filling factor to $f \leqslant 0.025$. This is an upper limit to $f$. In some spectra the wings of the lines are even weaker, but smaller values for $f$ might be beyond the validity of the approximations used in the formalism. We used $f=0.025$ for all models for all epochs.

\subsubsection{The Individual Spectral Fits}

Spectrum 1994. Of the four spectra, this one corresponds to the coolest temperature. N III $1750 \AA$ blend is clearly seen and we use the ratio N III 1750/N IV $1718 \AA$ as a temperature diagnostic. The optical part of the spectrum shows N III $4640 \AA$ line which is also well reproduced. In the low-resolution $I U E$ spectrum, the observed Fe IV lines in the 1600-1700 $\AA$ are much weaker than predicted. The mass-loss rate was fixed mainly by N IV] 1486 but is consistent with the other UV emission lines. Taking into account the clumping, our derived $\dot{M}$ is in reasonable agreement with that derived in Koenigsberger et al. (1998b) for the same observed spectrum. A likely explanation for this discrepancy is that the model used in that study considered only hydrogen and helium, thus missing the important influence of the line blanketing.

Spectrum 2000. The temperature of the star is low enough to show a measurable N III $4640 \AA$ A. We used that line to restrict the upper limit of the temperature. The mass-loss rate was constrained by NIV] $1486 \AA$ and further check with the Fe v UV complex. The model also reproduces well the optical He I and He II lines.

Spectrum 2002. The spectrum of this epoch does not include the optical wavelengths. We set the temperature based on the Fe VI/Fe V features observed between $1250 \AA$ and $1400 \AA$ and $1420 \AA$ and $1480 \AA$, respectively. The mass-loss rate is derived from N IV] 1486 and the P v 1118-28 A doublet.

Spectrum 2009. The temperature was limited from below by the absence of N III $4640 \AA$. This line is present in the previous epochs but is weak in this last spectrum. In addition we used the $\mathrm{Fe}$ v/Fe IV UV complex.
The uncertainties in the results obtained from the model fits are difficult to estimate due to the large number of free parameters which are not completely independent. However, the models show that changing $\mathrm{T}\left(\tau_{\text {Ross }}=10\right)$ by more than $\sim 2000 \mathrm{~K}$ causes changes in the computed spectrum that make it noticeably different from the observations. In an analogous manner, the uncertainty in the mass-loss rate can be estimated to be $30 \%$. The uncertainty in the luminosity due to the uncertainties in the flux calibration and in the $\mathrm{S} / \mathrm{N}$ of the spectra is $\lesssim 20 \%$. The absolute uncertainty in the parameters is much larger due to unclear correlations between them which are difficult to quantify. We minimize these errors by making our models for the different epochs consistent so the differences in the parameter values are expected to reflect real changes in the stellar physics.

\subsection{Results}

Table 6 summarizes the parameters derived from the models of Star A in the epochs 1994, 1999, 2002, and 2009 as well as the model of Star $C$. The comparison between the observations and the calculated spectra are shown in Figures 11-15.

Three different radii are listed in Table 6: (1) $R_{10}$, which is the radius at which the Rosseland optical depth $\tau_{\text {Ross }}=10$, and it is this reference radius at which the gravity acceleration is $\log g$ and where the temperature $T_{10}$ is given by the CMFGEN model; (2) $R_{s}$, which is the radius at the sonic point, were the optical depth and temperature are, respectively, $\tau_{s}$ and $T_{s}$; we define the photosphere as that region of the star with $r<R_{s}$ and the wind where $r>R_{s}$; (3) $R_{2 / 3}$, which is the radius of the continuum-forming region, where $\tau_{\text {Ross }}=2 / 3$, and here $T_{\text {eff }}=T\left(R_{2 / 3}\right)$.

The most outstanding results derived from these models are as follows.

1. The luminosity of Star A is higher during the 1994 eruptive event than at later epochs. This is consistent with the results obtained by Drissen et al. (2001) from a spectrum take on 1994 September 21. Their luminosity is even higher than our value for the spectrum obtained on 1994 

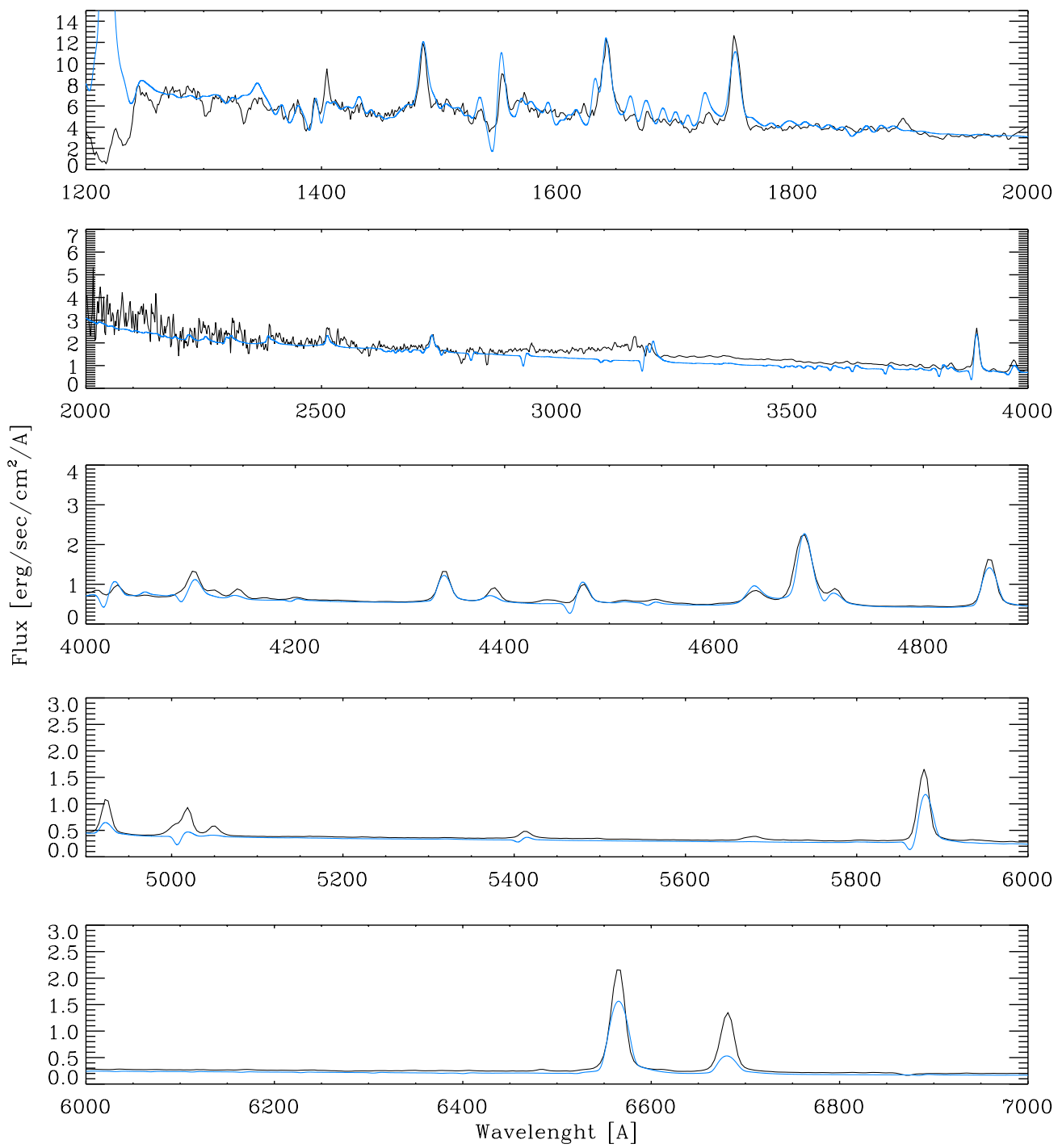

Figure 13. Comparison between the spectrum obtained on 1994 December 30 (black) and the sum of the current best model for Star A at this epoch and Star C model (blue). The singlet He I lines are underestimated while the triplet lines are well reproduced.

December 31. The luminosity is lowest in 2000 April, and then rises slightly in 2002 remaining at the same level until 2009. Thus, we are now able to confirm that the 1994 eruption is an event which did not occur at constant luminosity.

2. Star A's mass-loss rate is a factor of $\sim 4$ larger in 1994 than at later epochs. The larger luminosity most likely plays an important role in driving the larger mass-loss rate.

3. The radius at $\tau_{\text {Ross }}=2 / 3, R_{2 / 3}=124 R_{\odot}$, is larger in 1994 than at any other epoch, and it is very much larger than $R_{s}=21.5 R_{\odot}$, the radius at the sonic point in 1994 . The continuum-forming region is very extended. Figure 16 shows the temperature structure of the 1994 model where one can also see that due to the larger wind density, the temperature is significantly higher throughout the wind. It is also quite remarkable that the temperature at the sonic point is $T=10^{5} \mathrm{~K}$. This high temperature is due to the fact that radiation is trapped within the extended continuum emitting region.

4. $R_{2 / 3}$ declines after 1994 reaching $28 R_{\odot}$ in 2009 . This value is larger than the size of the observed continuum-emitting region of Star A in 1979 (22-25 $R_{\odot}$; Perrier et al. 2009), consistent with the notion that Star A has still not recovered its minimum state of activity. The decrease in $R_{2 / 3}$ reflects the shrinking size of the continuum forming region as the density drops. Note, however, that $R_{s}=19.5 R_{\odot}$ in 2009 epoch, indicating that the continuum formation region is still rather extended.

5. The most significant change in $R_{S}$ occurred in the 2000 epoch, at which time it was larger than during the other epochs. This is consistent with the long-term behavior of the optical light curve which peaked at around the 2000 epoch and with the notion that the long-term activity cycle and the brief eruptions of 1993-1994 seem to be separate phenomena (Koenigsberger et al. 2010).

6. There is a $30 \%$ variation in $\dot{M}$ between 2000 and 2002 and it remains relatively constant thereafter. Although it is tempting to suggest a declining trend, the variation lies within the uncertainties of the model results. Hence, we tentatively conclude that $\dot{M}$ is approximately constant over the timescale 2000-2009. This leads to the conclusion that the observed spectral changes during this timescale result primarily from the declining wind density, caused by the increasing wind velocity. Hence, we are now in the 

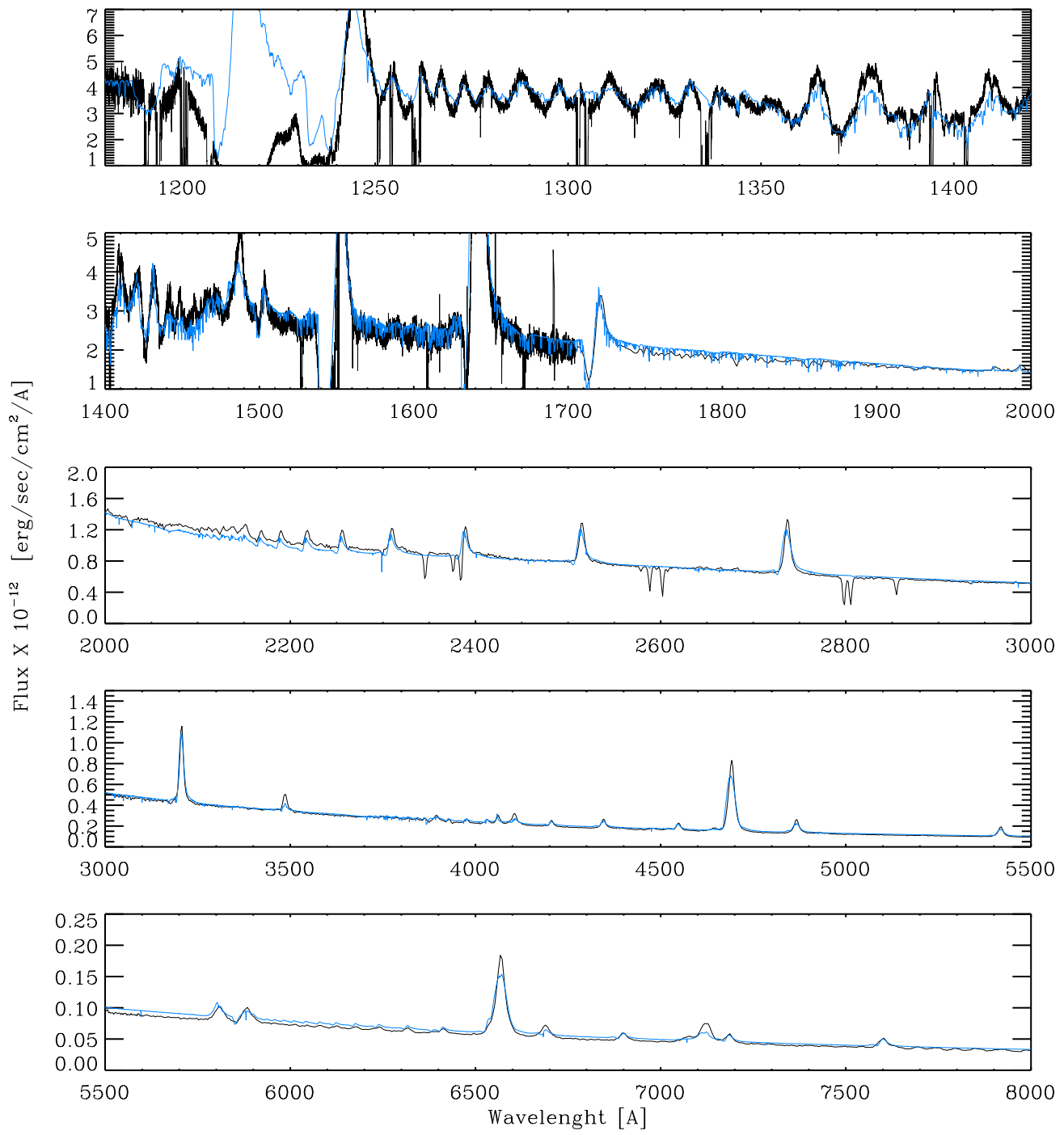

Figure 14. As in Figure 13 but for the 2000 April epoch.

possibility of understanding the relation between the flux in N IV] $1486 \AA$ and the terminal wind speed (Figure 5). Based on the models we can conclude that the reduction of the post-eruption line intensities is caused mainly by the increase of $V_{\infty}$. Assuming that $V_{\infty} / V_{\text {esc }}$ is constant (Figure 17) one can conclude that the changes in $V_{\infty}$ are caused by a decreasing radius of the star (Table 6). The relation between N IV] $1486 \AA$ and the terminal velocity is maintained during the pre-eruption epochs and it follows the same general trend (Koenigsberger et al. 2010) but in the opposite direction. $V_{\infty}$ decreases while N IV] $1486 \AA$ increases which points to an increase in the stellar radius. This leads us to conclude that the S Dor-type $\sim 40$ year variability timescale of Star $A$ is due to variations in its radius.

The S Dor-type variability of Star $A$ is similar to that of AG Car (Groh et al. 2009) and S Dor (Lamers 1995). Excluding the 1994 eruption event, the maximum of the optical brightness in HD 5980 was attained between 1996 and 2000. At that time, the terminal wind velocity and the temperature were lower than in 2009, when the visual brightness was approaching its minimum. In this sense, during its long term variability, Star A behaves similarly to AG Car and S Dor. Lamers (1995) explained this variability as a pulsation of $10^{-3}$ to $10^{-2}$ of the stellar mass in S Dor, and Groh et al. (2009) reached the same conclusion for AG Car. Lamers (1995) suggested that the larger radius decreases the gravity acceleration and increases the scale height which leads to larger mass loss. He also suggested that the luminosity of the star should be lower during the maximum of the optical brightness due to the work the star must perform to lift the upper layers. In agreement with this prediction we detected a small difference in the luminosity in 2000 (close to maximum) and 2009 (close to minimum) epochs which add weight to the suggestion that S Dor variability of Star A is caused by a pulsation. The exact physical mechanism causing the pulsation is unclear but now that we have three objects showing similar effects, the question arises as to whether this may be a common phenomenon in this class of objects.

\section{THE 1994 ERUPTION AND THE BI-STABILITY JUMP OF A SECOND KIND}

The results presented in Table 6 indicate that the wind structure of Star A in 1994 was significantly different from its wind structure during the other three epochs. This difference is best illustrated in Figure 16 where we plot the temperature structure derived from the spectra of 1994 and 2009. The 

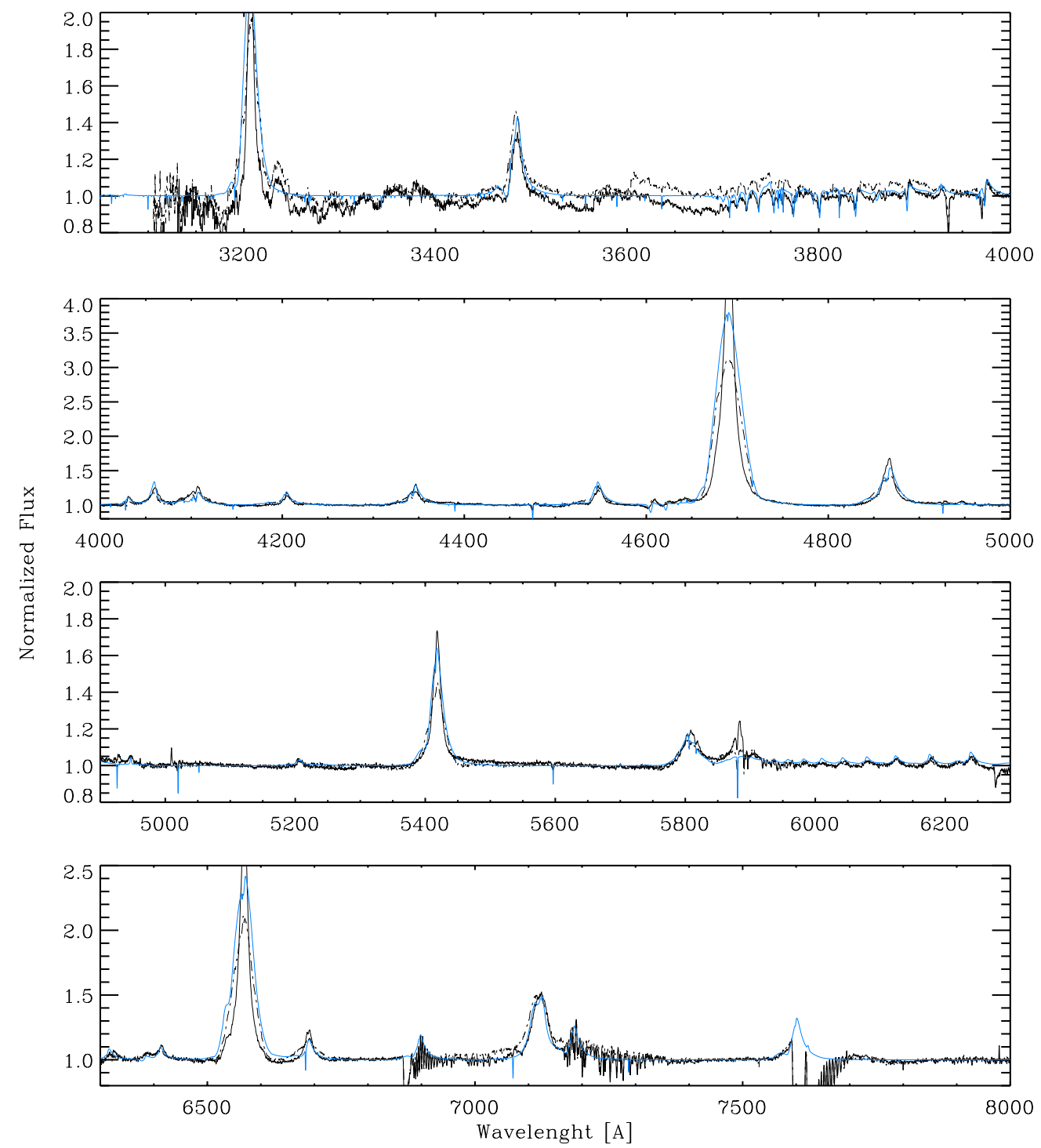

Figure 15. As in Figure 11 but for the optical region. The spectrum is affected by the presence of strong telluric absorption lines in $6850 \AA$, $7200 \AA$, and $7550 \AA$. The observed profile of $\mathrm{He}$ II $4686 \AA$ and $\mathrm{H}_{\alpha}$ is much narrower than the model spectrum. Breysacher et al. (1982) showed that He II $4686 \AA$ changes its profile with the orbital period being narrower at the eclipses. The model shows that the linewidth outside the eclipse corresponds to the UV P Cyg lines and there is missing emission during the eclipse. The interpretation of this effect requires a reliable model for Star B which will be treated in a forthcoming paper.

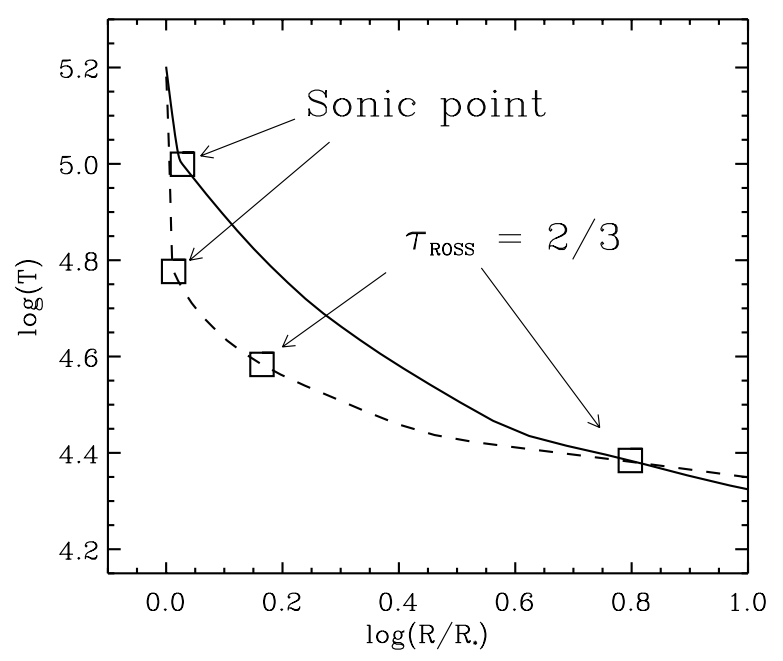

Figure 16. Temperature distribution in the 1994 (solid line) and 2009 (dashed line) models. The positions of the sonic point and the continuum forming regions $\left(\tau_{\text {Ross }}=2 / 3\right)$ are indicated. The lower mass-loss rate in the 2009 model moves the continuum-forming region inward. temperature is higher throughout most of the wind in 1994 than in 2009, even though the observed 1994 spectrum looks cooler. This is a consequence of the higher density in 1994 which makes the wind more optically thick.

The velocity structures are also different, in particular, the relation between the terminal wind velocity, $V_{\infty}$ and the escape velocity $V_{\text {esc }}$, which is defined as

$$
V_{\mathrm{esc}}=\left(\frac{G M(1-\Gamma)}{R_{10}}\right)^{1 / 2}
$$

where $\mathrm{M}$ is the stellar mass assumed to be $90 M_{\odot}$. The ratio between gravity acceleration and the radiation pressure $\Gamma=g_{e l} / g_{\text {grav }}$ takes into account only the radiation pressure caused by electron scattering (Lamers \& Cassinelli 1999). We calculate $V_{\text {esc }}$ using $\Gamma$ as determined by the CMFGEN model at $R_{10}$. Figure 17 shows $V_{\infty}$ plotted against $V_{\text {esc }}$. The values for the three post-eruption epochs (2000, 2002, and 2009) lie close to a single straight line, the slope of which is $\sim 2.3$. The wind during the 1994 epoch is different. In this case, $V_{\infty} / V_{\text {esc }} \sim 1.2$. 


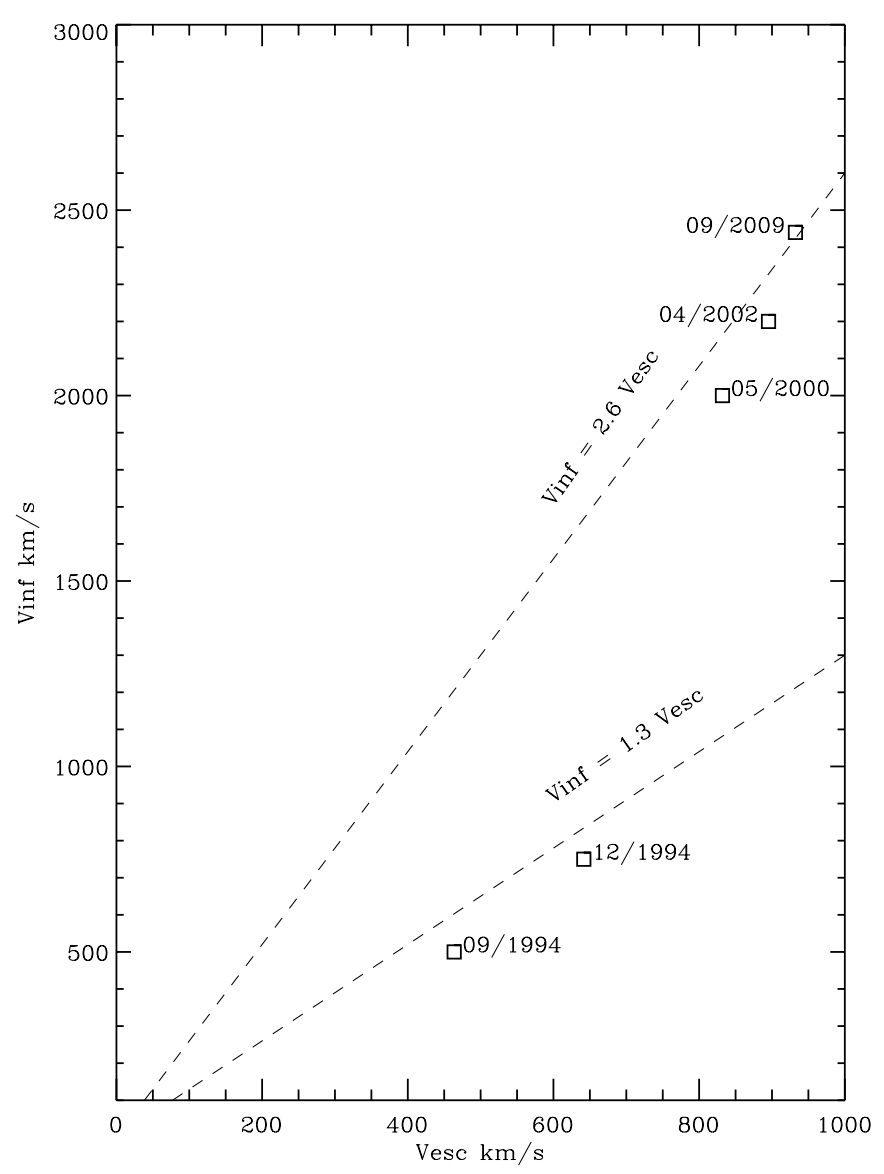

Figure 17. Relation between $V_{\infty}$ and $V_{\text {esc }}$ at different epochs.

The velocities obtained by the Drissen et al. (2001) for 1994 September lie along the same relation.

The change in the $V_{\infty} / V_{\text {esc }}$ relation together with the cooler effective temperature of the 1994 spectrum are strongly suggestive of a phenomenon associated with the bi-stability jump (Lamers et al. 1995). The bi-stability jump occurs when the temperature in the inner wind decreases to a value at which the ionization fraction of $\mathrm{Fe}^{2+}$ increases leading to changes in the line opacities and a changes of the driving force, higher mass-loss rate and reduce in $V_{\infty} / V_{\text {esc }}$ from $\sim 2.6$ to 1.3 (Vink et al. 1999). This occurs at $T_{\text {eff }} \sim 21000 \mathrm{~K}$.

This is remarkably similar to what we find for HD 5980. There is, however, a major difference between the cause of the bi-stability jump observed in $\mathrm{O}$ and $\mathrm{B}$ stars and the phenomenon that our models indicate is occurring in HD 5980. As was shown by Lamers \& Cassinelli (1999) and Vink et al. (1999), the massloss rate is defined by the force below the sonic point. In the optically thin $\mathrm{O}$ and B star's winds the sonic point is above the photosphere, thus the condition in it can be described by the effective temperature. On the other hand, the sonic point of the optically thick wind of HD 5980 is located below the pseudo photosphere (Figure 16). In the 1994 model, the point at which the temperature $T \sim T_{\text {eff }} \sim 21000 \mathrm{~K}$ lies at $\sim 6 R_{S}$ far above the sonic point. Thus, changes in the ionization structure at a distance of $\sim 6 R_{s}$ have little effect on the overall wind structure.

In search of an alternative cause of the jump we followed the reasoning of Lamers \& Nugis (2002) and Gräfener \& Hamann (2008). These authors showed that the WR winds below the sonic point are driven not by the line force but rather by the radiation pressure due to continuum opacities. We applied that idea to Star A's wind. In Table 6 we see that the optical depth of the sonic point $\tau_{s}>1$ for all four epochs, indicating that the wind is driven primarily by the continuum force. Nugis \& Lamers (2002), and Gräfener \& Hamann (2008) showed that in order to drive a WR-type wind, the continuum opacity (sum of the true continuum opacities and the pseudo continuum of overlapping lines) has to be dominated by one of the bumps in the iron opacity. Gräfener \& Hamann (2008) showed that the required high mass-loss rate is obtained only if the temperature at the sonic point is either in the 30-70 kK range ("cool" bump) or around $160 \mathrm{kK}$ ("hot" bump). Table 6 shows that all of the post eruption models have $T_{s} \sim 48-50 \mathrm{kK}$, well within the first of these temperature intervals. One can thus expect that at almost constant luminosity the mass-loss rate will be almost constant, which is indeed the result from our models (Table 6). During the eruption the luminosity of the star increased. The higher luminosity heated the inner part of the wind changing the opacity contributors from those of the "cool" bump to those of the "hot" bump, thus changing the driving force and therefore the mass-loss rate. Our model for 1994 December gives $T_{s}=$ $100 \mathrm{kK}$ (Table 6). This temperature is too high for the "cool" iron opacity bump but too cool for the "hot" bump at $160 \mathrm{kK}$. Gräfener \& Hamann (2008) suggested that winds with $T_{s}$ in the intermediate temperature range are unstable. We speculate that at the onset of the eruption $T_{s}$ was high enough and the very dense wind was driven by the high temperature iron bump. As the luminosity decreased, the cooler wind became unstable and it rapidly switched back to the "cool" iron bump driving with $T_{s}$ around $50 \mathrm{kK}$.

We suggest that a bi-stability jump of a second kind operates in Star A. The sudden change in the wind properties is due to a transition from the "hot" to the "cool" Fe-bump opacity regimes mechanism that was shown by Lamers \& Nugis (2002) and Gräfener \& Hamann (2008) to be needed to drive the winds of WR stars.

Our models show that the increased luminosity can indeed change $T_{s}$, changing the entire wind structure during the eruption. Figure 16 shows the change in the temperature structure of the wind in the 1994 and 2009 models. In the less dense wind of 2009, the continuum forming region has moved further in and $T_{\text {eff }}$ increased. The sonic point is at a similar radius but due to the overall smaller temperature gradient it is significantly cooler. In addition to the changes in the temperature, the value of $\Gamma$ changes from $\sim 0.75$ during the eruption to $\sim 0.53$ afterward. A similar result was found by Smith et al. (2004). These authors showed that when the star approaches the Eddington limit, the optical depth of the sonic point increases and it moves below the photosphere. The increased luminosity of Star A moved it closer to the Eddington limit and the wind reacted in a similar manner. Furthermore Gräfener \& Hamann (2008) found that the WN stars with SMC chemical composition require $\Gamma>0.75$ to maintain a high mass-loss rate. In addition the Vink et al. (2011) models show a transition from $\mathrm{O}$ star wind to WR wind when $\Gamma$ increases above 0.7. Based on these considerations we can conclude that the wind during the eruption is governed by different forces than the wind in the post-eruption epochs. We speculate that during the eruption, the high luminosity heats the inner part of the wind, driving much higher mass loss trough the "hot" iron opacity bump. After the eruption the temperature at the sonic point drops making the wind unstable. The mass-loss rate drops, which causes further cooling of the sonic point until it reaches the "cool" iron bump where the wind stabilizes again and the 
star continues its long-term variability but with a restructured wind.

Hence, we suggest that for the dense winds such as those in HD 5980, there exists a second type of bi-stability jump than that proposed by Lamers et al. (1995). It is caused by transition between the "hot" and "cold" iron opacity bump and is correlated mainly with the stellar luminosity rather than with the effective temperature. Smith et al. (2004) discussed the position of the sonic point in the typical LBVs. They showed that for the derived parameters of most of the LBVs, the sonic point is located above the photosphere and thus their winds change according to the "classical" bi-stability jump. Here we speculate that the most luminous LBVs, such as Star A, have optically thick winds with pseudo photospheres above their sonic points even on the hotter side of the S Dor instability strip. Thus their excursion to the cool branch of the strip is caused by a second kind of bi-stability jump related to the changes in their continuum opacities.

\section{CONCLUSIONS}

HD 5980 is a relatively nearby system in which processes involving eruptive phenomena and massive star evolution in binary systems in a low metallicity environment can be studied. In this respect, is important to gain an understanding of the phenomena that it has presented over the past few decades.

In this paper we present the results of the analysis of a collection of UV and optical spectra obtained between 1979 and 2009 and performed CMFGEN model fits to spectra of 1994, 2000, 2002, and 2009. The primary results are as follows. (1) The long term S Dor-type variability is associated with changes of the hydrostatic radius. We find that the radius of the eruptor at the sonic point was largest in the year 2000 $\left(R_{s}=24.3 R_{\odot}\right)$, coinciding with the long-term maximum in the visual light curve. The minimum value in our data set is in $2009\left(R_{s}=19.6 R_{\odot}\right)$. (2) The 1994 eruption involved changes in the eruptor's bolometric luminosity and wind structure. The luminosity was larger by at least $50 \%$ with respect to the subsequent epochs and the temperature throughout most of the wind was significantly higher. The latter is a consequence of the very large optical depth which causes photons to be trapped within this wind region. At the same time, the velocity structure was one in which the relationship between the terminal wind velocity and the escape velocity $V_{\infty} / V_{\text {esc }} \sim 1.2$, contrary to other epochs in which this ratio was $\sim 2.3$. (3) The emissionline strength, the wind velocity and the continuum luminosity underwent correlated variations in the sense that a decreasing $V_{\infty}$ was associated with increasing emission line and continuum levels. These correlations can be understood in the context of the increasing size of the hydrostatic stellar radius. (4) The spectrum of the third star in the system $($ Star $C)$ is well fit by a $T_{\text {eff }}=$ $32 \mathrm{~K}$ model atmosphere with SMC chemical abundances. The abundances of the eruptor, Star A, show He and N enhancements, roughly consistent with the $\mathrm{CNO}$ cycle equilibrium values. A similar composition was obtained by Groh et al. (2009) for AG Car and Hillier et al. (2001) for $\eta$ Car.

For all epochs, the wind of the erupting star is optically thick at the sonic point and is thus driven mainly by the continuum opacity. We speculate that the wind switches between two stable regimes driven by the "hot" (during the eruption) and the "cool" (post-eruption) iron opacity bumps as defined by Lamers \& Nugis (2002) and Gräfener \& Hamann (2008), and thus the wind may undergo a bi-stability jump of a different nature from that which occurs in OB stars.
We thank Alfredo Díaz and Ulises Amaya for computing assistance. This research was supported under UNAM/DGAPA/PAPIIT grants IN106798 and IN123309 and CONACYT grants 48929 and 83016. L.N.G. acknowledges the support from CONACyT grant 141530. R.H.B. acknowledges partial support from ULS DIULS grant, and D.J.H. acknowledges support from grant HST-GO-11623.01-A and HST-GO11756.01. The HST is operated by the STScI, under contract with AURA.

\section{REFERENCES}

Barbá, R. H., Morrell, N. I., Niemela, V. S., et al. 1996, Rev. Mex. Astron. Astrofis. Ser. Conf., 5, 85

Barbá, R. H., \& Niemela, V. S. 1994, IAU Circ., 6099, 1

Barbá, R. H., Niemela, V. S., Baume, G., \& Vazquez, R. A. 1995, ApJ, 446, L23 Barbá, R. H., Niemela, V. S., \& Morrell, N. I. 1997, in ASP Conf. Ser. 120 , Luminous Blue Variables: Massive stars in Trnsition, ed. A. Nota \& H. Larmers (San Francisco, CA: ASP), 238

Breysacher, J. 1997, in ASP Conf. Ser. 120, Luminous Blue Variables: Massive Stars in Transition, ed. A. Nota \& H. Lamers (San Francisco, CA: ASP), 227 Breysacher, J., Moffat, A. F. J., \& Niemela, V. 1982, ApJ, 257, 116

Breysacher, J., \& Perrier, C. 1980, A\&A, 90, 207

Cardelli, J. A., Clayton, G. C., \& Mathis, J. S. 1989, ApJ, 345, 245

Colina, L., Bohlin, F., \& Castelli, F. 1996, Instrument Science Report CAL/ SCS-008

Crowther, P. A., Hillier, D. J., Evans, C. J., et al. 2002, ApJ, 579, 774

Drissen, L., Crowther, P. A., Smith, L. J., et al. 2001, ApJ, 545, 484

Dwarkadas, V. V. 2011, MNRAS, 412, 1639

Eenens, P. R. J., \& Williams, P. M. 1994, MNRAS, 269, 1082

Foellmi, C., Koenigsberger, G., \& Georgiev, L. 2008, RevMexAA, 44, 3

Georgiev, L. N., \& Koenigsberger, G. 2004, A\&A, 423, 267

Gräfener, G., \& Hamann, W.-R. 2008, A\&A, 482, 945

Grevesse, N., Asplund, M., \& Sauval, A. J. 2007, Space Sci. Rev., 130, 105

Groh, J. H., Hillier, D. J., Damineli, A., et al. 2009, ApJ, 698, 1698

Guzik, J. A., Cox, A. N., \& Despain, K. M. 2005, in ASP Conf. Ser. 332,

The Fate of the Most Massive Stars, ed. R. Humphreys \& K. Stanek (San Francisco, CA: ASP), 263

Herald, J., \& Bianchi, L. 2011, MNRAS, in press

Heydari-Malayeri, M., Rauw, G., Esslinger, O., \& Beuzit, J.-L. 1997, A\&A, 322,554

Hilditch, R. W., Howarth, I. D., \& Harries, T. J. 2005, MNRAS, 357, 304

Hillier, D. J., Davidson, K., Ishibashi, K., \& Gull, T. 2001, ApJ, 553, 837

Hillier, D. J., Lanz, T., Heap, S. R., et al. 2003, ApJ, 588, 1039

Hillier, D. J., \& Miller, D. 1998, ApJ, 496, 407

Hunter, I., Dufton, P. L., Smartt, S. J., et al. 2007, A\&A, 466, 277

Jones, A., \& Sterken, C. 1997, J. Astron. Data, 3, 3

Kashi, A., \& Soker, N. 2010, ApJ, 723, 602

Koenigsberger, G. 2004, RevMexAA, 40, 107

Koenigsberger, G., Auer, L. H., Georgiev, L., \& Guinan, E. 1998a, ApJ, 496, 934

Koenigsberger, G., Fullerton, A., Massa, D., \& Auer, L. H. 2006, AJ, 132, 1527

Koenigsberger, G., Georgiev, L., Barbá, R., et al. 2000, ApJ, 542, 428

Koenigsberger, G., Georgiev, L., Hillier, D. J., et al. 2010, AJ, 139, 2600

Koenigsberger, G., Georgiev, L., Peimbert, M., et al. 2001, AJ, 121, 267

Koenigsberger, G., Guinan, E., Auer, L., \& Georgiev, L. 1995, ApJ, 452, L107

Koenigsberger, G., Kurucz, R., \& Georgiev, L. 2002, ApJ, 581, 598

Koenigsberger, G., Moffat, A. F. J., \& Auer, L. H. 1987, ApJ, 322, L41

Koenigsberger, G., Moffat, A. F. J., St-Louis, N., et al. 1994, ApJ, 436, 301

Koenigsberger, G., Pena, M., Schmutz, W., \& Ayala, S. 1998b, ApJ, 499, 889

Koenigsberger, G., Shore, S., Guinan, E., \& Auer, L. 1996, Rev. Mex. Astron. Astrofis. Ser. Conf., 5, 92

Lamers, H. J. G. L. M. 1995, in ASP Conf. Ser. 83, IAU Colloq. 155: Astrophysical Applications of Stellar Pulsation, ed. R. S. Stobie \& P. A. Whitelock (San Francisco, CA: ASP), 176

Lamers, H. J. G. L. M., \& Cassinelli, J. P. 1999, Introduction to Stellar Winds (Cambridge: Cambridge Univ. Press)

Lamers, H. J. G. L. M., \& Nugis, T. 2002, A\&A, 395, L1

Lamers, H. J. G. L. M., Snow, T. P., \& Lindholm, D. M. 1995, ApJ, 455, 269

Maíz-Apellaniz, J., \& Bohlin, R. C. 2005, Space Telescope Science Institute, Instrument Science Report STIS 2005-01

Moffat, A. F. J., Marchenko, S. V., Bartzakos, P., et al. 1998, ApJ, 497, 896

Morisset, C., \& Georgiev, L. 2009, A\&A, 507, 1517

Nichols, J. S., \& Linsky, J. L. 1996, AJ, 111, 517 
Niemela, V. S. 1988, in ASP Conf. Ser. 1, Progress and Opportunities in Southern Hemisphere Optical Astronomy, ed. V. M. Blanco \& M. M. Phillips (San Francisco, CA: ASP), 381

North, P., Gauderon, R., Barblan, F., \& Royer, F. 2010, A\&A, 520, A74

Nugis, T., \& Lamers, H. J. G. L. M. 2002, A\&A, 389, 162

Owocki, S., \& van Marle, A. J. 2008, in IAU Symp. 250, Massive Stars as Cosmic Engines, ed. A. Maeder, G. Meynet, S. Ekström, R. Hirschi, \& C. Gregory (Cambridge: Cambridge Univ. Press), 71

Peimbert, M., Peimbert, A., \& Ruiz, M. T. 2000, ApJ, 541, 688

Perez, M. 1992, NASA IUE Newsletter, 48, 76

Perrier, C., Breysacher, J., \& Rauw, G. 2009, A\&A, 503, 963

Pojmanski, G. 2002, Acta Astron., 52, 397
Prinja, R. K., Barlow, M. J., \& Howarth, I. D. 1990, ApJ, 361, 607

Schmutz, W., Hamann, W.-R., \& Wessolowski, U. 1989, A\&A, 210, 236

Schweickhardt, J. 2000, PhD thesis, Ruprecht-Karls-Universität, Heidelberg Smith, N. 2008, in IAU Symp. 250, Massive Stars as Cosmic Engines, ed. A. Maeder, G. Meynet, S. Ekström, R. Hirschi, \& C. Gregory (Cambridge: Cambridge Univ. Press), 193

Smith, N., Vink, J. S., \& de Koter, A. 2004, ApJ, 615, 475

Sterken, C., \& Breysacher, J. 1997, A\&A, 328, 269

van Marle, A. J., Langer, N., \& García-Segura, G. 2007, A\&A, 469, 941

Vink, J. S., de Koter, A., \& Lamers, H. J. G. L. M. 1999, A\&A, 350, 181

Vink, J. S., Muijres, L. E., Anthonisse, B., et al. 2011, A\&A, 531, 132 